\title{
A Methodological Review of Piezoelectric Based Acoustic Wave Generation and Detection Techniques for Structural Health Monitoring
}

\author{
Zhigang Sun, ${ }^{1}$ Bruno Rocha, ${ }^{2}$ Kuo-Ting Wu, ${ }^{1}$ and Nezih Mrad $^{3}$ \\ ${ }^{1}$ Energy, Mining and Environment Portfolio, National Research Council Canada, Boucherville, QC, Canada J4B $6 Y 4$ \\ ${ }^{2}$ Aerospace Portfolio, National Research Council Canada, Ottawa, ON, Canada K1A OR6 \\ ${ }^{3}$ Operational Analysis and Systems Integration Support, Defence R\&D Canada-Atlantic, Department of National Defence, \\ Ottawa, ON, Canada K1A OK
}

Correspondence should be addressed to Zhigang Sun; zhigang.sun@cnrc-nrc.gc.ca

Received 21 August 2012; Revised 19 December 2012; Accepted 25 December 2012

Academic Editor: Victor Giurgiutiu

Copyright (C) 2013 Zhigang Sun et al. This is an open access article distributed under the Creative Commons Attribution License, which permits unrestricted use, distribution, and reproduction in any medium, provided the original work is properly cited.

\begin{abstract}
Piezoelectric transducers have a long history of applications in nondestructive evaluation of material and structure integrity owing to their ability of transforming mechanical energy to electrical energy and vice versa. As condition based maintenance has emerged as a valuable approach to enhancing continued aircraft airworthiness while reducing the life cycle cost, its enabling structural health monitoring (SHM) technologies capable of providing on-demand diagnosis of the structure without interrupting the aircraft operation are attracting increasing R\&D efforts. Piezoelectric transducers play an essential role in these endeavors. This paper is set forth to review a variety of ingenious ways in which piezoelectric transducers are used in today's SHM technologies as a means of generation and/or detection of diagnostic acoustic waves.
\end{abstract}

\section{Introduction}

Piezoelectric transducers are omnipresent in modern human life, from a kitchen gas lighter to popular gadgets such as smart phones and tablet computers, from submarine sonar to medical echography systems, and from electric watches to more dedicated measurement devices such as accelerometers for aircraft vibration monitoring and pressure sensors in jet engines for engine operation monitoring. Piezoelectric transducers can be used either as an actuator, for example, as the ultrasonic motor in a camera lens, or as a sensor, as in the case of an ultrasonic fish finder. These transducers can be used stand-alone, as are the case of conventional ultrasonic nondestructive evaluation (NDE) and nondestructive inspection (NDI) sensors, or be an integrated part of microelectromechanical systems (MEMS). The piezoelectric elements can also be used as harvesters of mechanical vibration energy.

From an acoustic point of view, there is no difference between SHM and conventional NDE/NDI since both rely on the same physics in the sense that in either case acoustic waves need to be generated and then detected, independently of the acoustic wave mode or method used. What truly differentiate an SHM approach from an NDE/NDI approach is that the former usually requires the sensing elements to be permanently mounted on or embedded in the structure to be evaluated while allowing the wave generation elements, if active wave generation is used by the inspection method, to be either permanently mounted or mobile, whereas in the latter case both wave generation and detection elements are mobile. The requirement of permanently installed SHM sensors, and in some cases, of acoustic wave generation devices, poses challenges and at the same time spurs the development of new sensing concepts which are not practicable in conventional $\mathrm{NDE} / \mathrm{NDI}$. The main objective of this paper is to review some of these unique concepts. Since the focus is on presenting the concepts, there will be no attempt to review all existing works which implement a same idea. Instead, the idea will be presented only through representative cases. It is important 


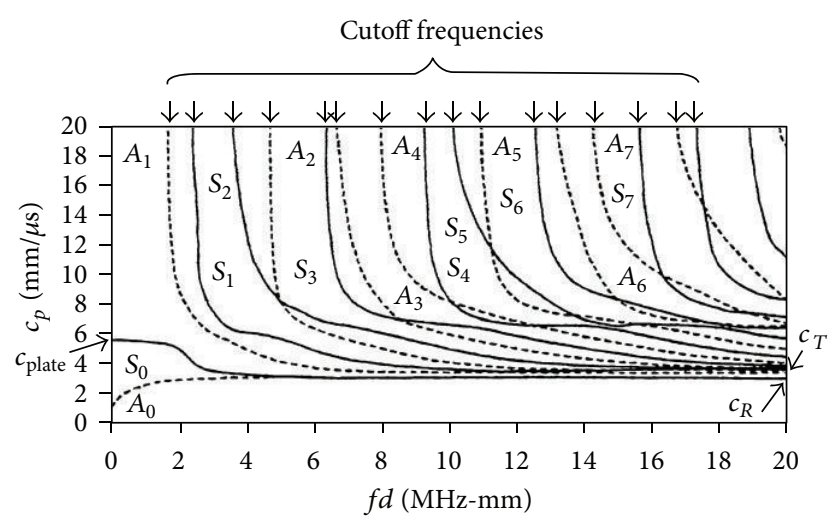

(a)

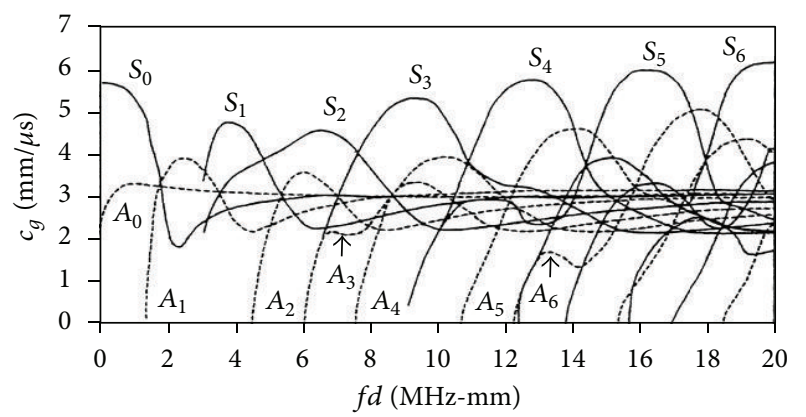

(b)

FIGURE 1: Dispersion curves for a traction free aluminum plate (the classic Lamb wave problem): (a) phase velocity dispersion curvesa and (b) group velocity dispersion curves $[1,2]$.

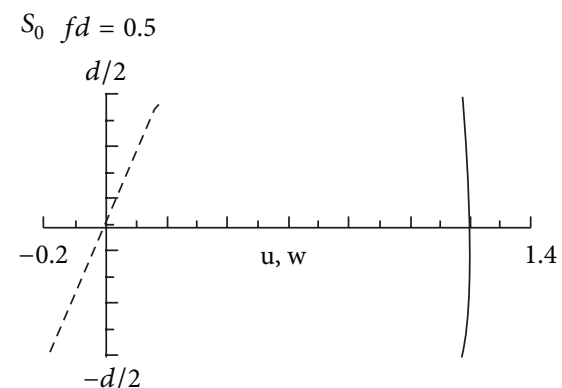

(a)

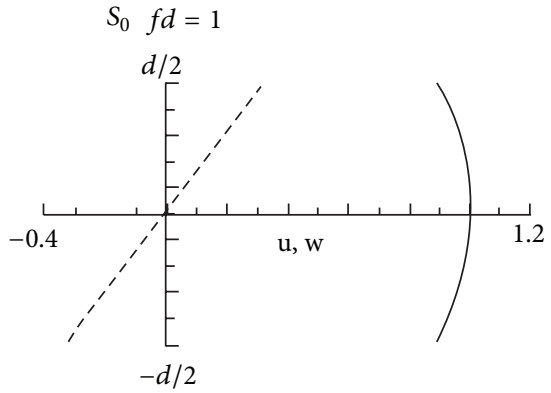

(b)

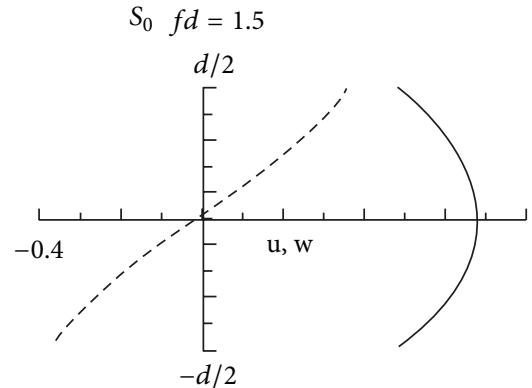

(c)

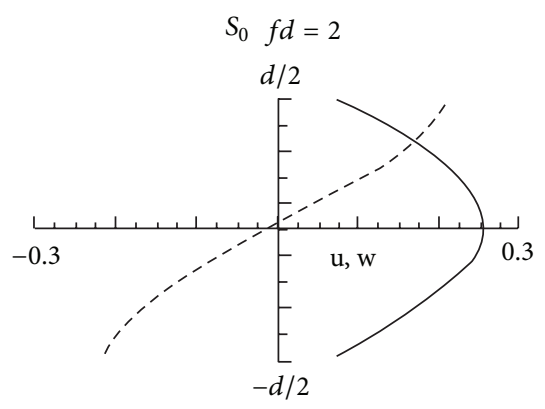

(d)

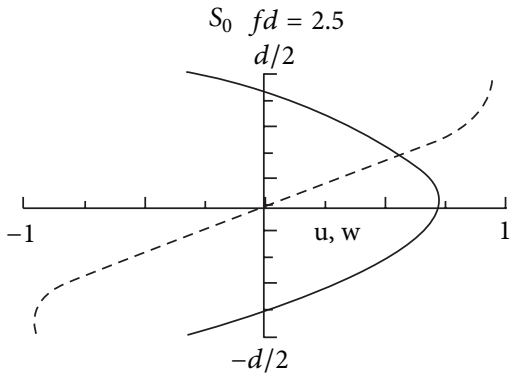

(e)

FIGURE 2: A wave structure for various points (particle displacements) for the $S_{0}$ mode in an aluminum plate, showing the in-plane (usolid line) and out-of-plane (w-dashed line) displacement profiles across the thickness of the plate, for different wave frequency and plate thickness products $[1,2]$.

to note that as sensors, piezoelectric transducers can be used in acoustic and nonacoustic based techniques. Piezoelectric pressure sensors, accelerometers, and structural electromechanical impedance sensors are examples of nonacoustic applications of piezoelectric transducers. Although these sensors are widely used in SHM, they are not within the scope of the present paper.

This paper is divided into three sections. The first section is dedicated to all-piezoelectric acoustic based approaches in which piezoelectric transducers are used for both generation and detection of acoustic waves. The second section presents hybrid approaches in which either piezoelectric transducers are used for acoustic wave generation (i.e., as exciters) and a nonpiezoelectric transducer based technique is used for wave detection or are used for wave detection whereas a nonpiezoelectric based technique is used for acoustic wave generation. The third section is devoted to wireless wave generation and detection techniques. 


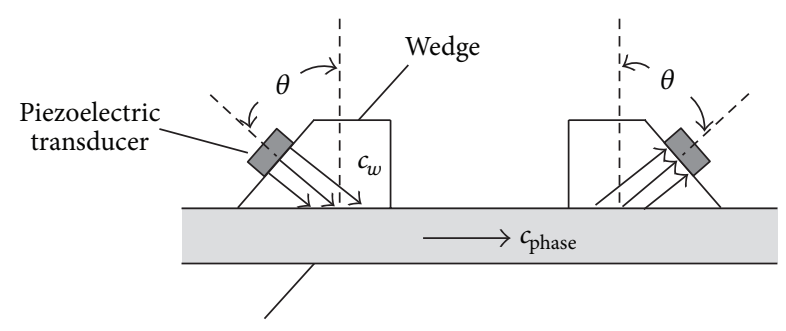

Thin-wall structure

FIGURE 3: Wave tuning using a wedge.

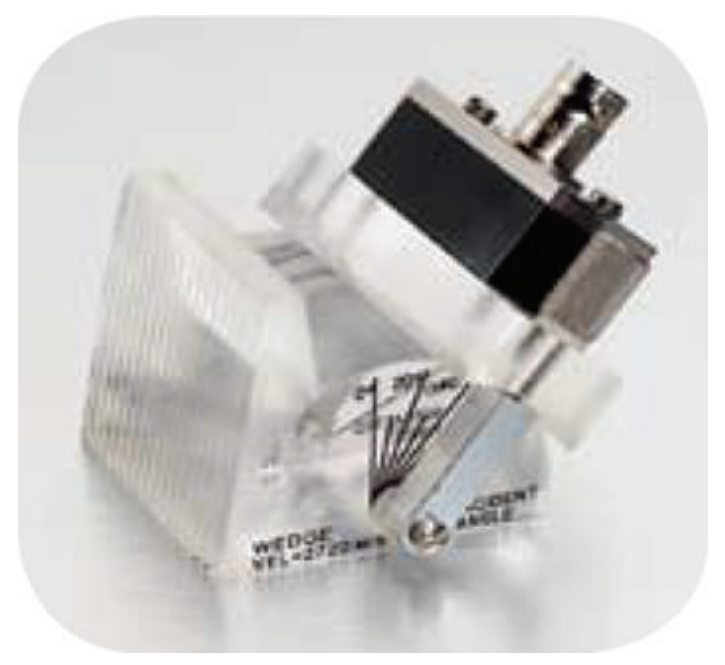

FIgURE 4: Olympus NDT variable angle beam wedge.

\section{All-Piezoelectric Approaches}

2.1. Some Basics. Thin-wall structural elements such as shells, plates, and pipes are building blocks of aircraft structures. While NDE/NDI techniques are in their majority applied in localized areas of the structure, employing through-thethickness wave propagation, SHM of aerospace structures via acoustic based techniques inevitably necessitates detection, and possibly also generation of acoustic waves propagating in thin-wall structures. Since these waves' propagation is confined in a thin layer and being guided by the two close-by and parallel (or quasiparallel) free surface boundaries in a thinwall member, they are usually called guided plate acoustic waves. Because of their confinement in a thin-wall structure and the close-by free surface boundary conditions, guided acoustic waves can travel over a long distance, even in high acoustic attenuating materials such as carbon fiber-reinforced composites (CFRP). This long distance reaching capability makes guided acoustic waves a powerful tool for evaluation of thin-wall structure integrity, broadening the inspection area and enabling the inspection of multiple sites in a thin-wall member with a limited number of transducerssmaller than the number of mounted transducers that would have to be used for the application of through-the-thickness propagating waves in a conventional NDE/NDI fashion.

There are two types of guided acoustic waves that can propagate in thin-wall structures. The first type is called Lamb waves to honor the British mathematician Horace Lamb who was the first to prove mathematically the existence of this type of waves, publishing a comprehensive analysis in 1917 [4]. Lamb waves can be decomposed into particle vibrations in two orthogonal (polarization) directions: one in line with wave propagation direction, also called in-plane direction, and another one perpendicular to both wave propagation direction and the wall surface, also called out-of-plane direction. Particle vibrations in these two directions are coupled. Depending on the vibration strength in each direction across wall thickness, different vibration patterns (modes) are formed. The second type of waves is horizontally polarized shear waves that vibrate in a direction perpendicular to the wave propagation direction and parallel to the wall surface. Owing to relative easiness of generation and detection with conventional piezoelectric acoustic transducers, Lamb waves are used more widely than shear horizontal $(\mathrm{SH})$ waves for NDE/NDI of structures.

By nature, guided acoustic waves are multimodal and frequency dispersive. This means that a plurality of particle vibration patterns (modes) can coexist in the same structure and each vibration pattern propagates at a different speed. Furthermore, the propagation speed and the vibration pattern of each mode can vary with the frequency of the wave. This multi-modal and dispersive nature can make a diagnostic signal so complicated that even an expert may not be able to extract relevant information about the structure integrity from the signal. For this reason, a great deal of applications in acoustic NDE/NDI has been focused on how to selectively generate and single out one or two simple modes of the generated acoustic waves and to make signal interpretation much less prohibitive while ensuring that the selected mode is sensitive to the defects or damage to detecta process often called "tuning." For example, the detection of through-the-thickness cracks with the pulse-echo method is considerably more successful with the use of the fundamental symmetric mode (usually denoted by $S_{0}$ ) than with the fundamental antisymmetric mode (usually denoted by $\left.A_{0}\right)$. On the other hand, $A_{0}$ mode seems to be better suited for the detection of delaminations, disbonds, and corrosion with pitch-catch acoustoultrasonics techniques [5]. The fundamental symmetric mode $\left(S_{0}\right)$ consists in a tractioncompression occurring parallel to the wave propagation direction and free surface boundaries and mid-plane of the thin-wall structural element where the wave propagates with the particles presenting a symmetric displacement with respect to the mid-plane of the structural component. The fundamental antisymmetric mode $\left(A_{0}\right)$ consists in a bending wave, with the main movement of the particles in the cross section of the thin-wall structural element being out of plane, that is, perpendicular to the wave propagation direction and to the free surface boundaries and mid-plane of the thin-wall structural member, and with the particles presenting an antisymmetric movement with respect to its mid-plane. The wave generation and receiving transducers should be "tuned" in order to enhance a specific mode while suppressing others.

The dispersive behavior of Lamb waves is represented by the dispersion curves, depicting the relationship between different wave characteristics which must be considered to 


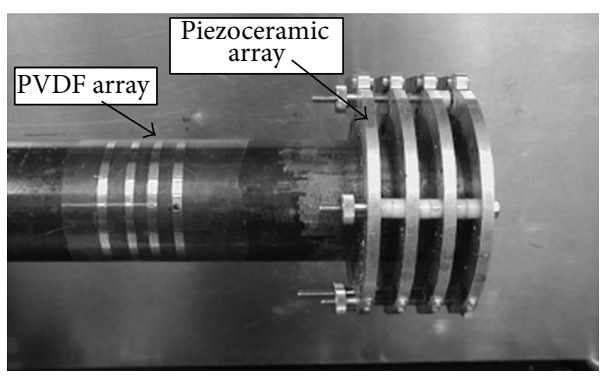

(a)

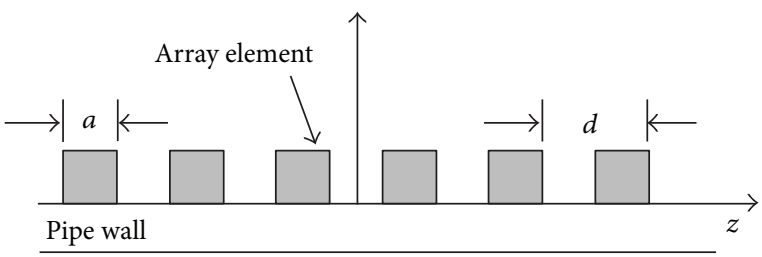

(b)

FIgURE 5: (a) Four-element PVDF and piezoceramic arrays and (b) array geometry [3].

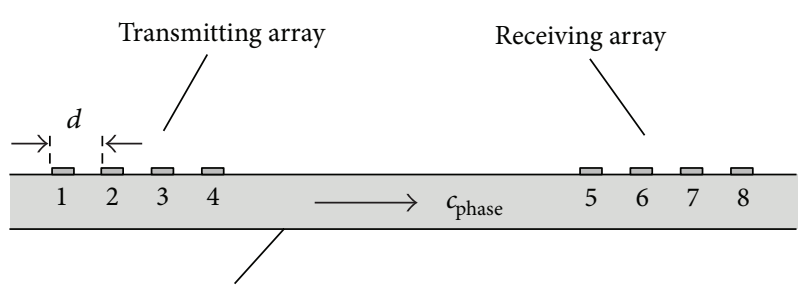

Thin-wall structure

FIGURE 6: Phased arrays for tuned wave generation and detection.

accomplish an effective tuning. For a thin-wall structural element with a certain thickness and made of a specific material, the dispersion curves present the dependence of wave phase velocity and group velocity on the wave frequency. The dependence of the waves' velocity on their frequency also results in a dependence of the wavelength on wave frequency. In Figure 1, the dispersion curves for the fundamental, 1st order, 2nd order, and so forth, symmetrical modes of Lamb waves, respectively, $S_{0}, S_{1}, S_{2}$, and so forth, are presented. In addition, the dispersion curves for the antisymmetrical modes of corresponding orders to $A_{0}, A_{1}, A_{2}$, and so forth are depicted. In these figures $f d$ represents the product of the frequency and the plate thickness. As depicted in Figure 2, for different Lamb wave modes, wave displacement profiles across the thin-wall member cross section are different. The first step in a tuning process consists, therefore, in the selection of the mode to apply (detect and potentially generate) in the inspection method. Afterwards, in an active technique, the excitation frequency of the wave generation transducer (exciter) must be selected. Generally, it is preferable to select an excitation frequency at which the following requirements are met as much as possible for the selected mode: (1) frequency dispersion of the phase velocity is minimal, that is; the slope in the phase velocity dispersion curve is minimal for a frequency range around the selected frequency (to minimize signal width spreading in time as a result of its propagation in space), at these frequencies the group velocity approximates well the phase velocity; (2) its group velocity differs sufficiently from those of other modes that coexist in the structure (to minimize the chance of observing the merge of different propagating wave modes); (3) wave displacement profile favors desired sensing of certain physical properties or defects; (4) wave displacement at the structure surface is easily detectable with the wave detection means. For example, if a longitudinal wave transducer is used, the displacement profile (f) in Figure 2 may be preferable to profile (a) due to much larger out-of-plane displacement at $f d=3$ and the fact that a longitudinal transducer is sensitive to out-of-plane wave motion. If a shear wave transducer polarized in the wave propagation direction is used, then profile (a) could be preferable to profile (d) because the shear wave transducer senses in-plane wave motion at the surface which is stronger in case (a). Depending on the application, used method and defects to detect, some of the above rules may not apply. For example, if the inspection method is based on the use of wave dispersion for structure integrity evaluation, then it may be preferable to excite the transducer at a frequency where the desired mode manifests significant frequency dispersion (i.e., a sharp slope in the dispersion curve). Some key characteristics of guided acoustics wave inspection and its applications in SHM are summarized in [6].

\subsection{Tuned Wave Excitation and Detection with Wedge Trans-} ducers. In the previous section, the importance of wave mode and wave frequency selections was explained in terms of the phase and group velocity dispersion curves and wave structure. In a practical application, the wave mode and frequency can be defined effortlessly by controlling the generated wave frequency through an electric excitation device. Furthermore, the selection of phase velocity can be realized by using a wedge. Let $c_{w}$ be the velocity of the acoustic waves (longitudinal or transverse) in the wedge, $c_{\text {phase }}$ the phase velocity of a desired wave mode at a selected frequency in the thin-wall structure, and $\theta$ the incident angle of the acoustic waves impinging on the structure, as depicted in Figure 3. According to Snell's law, acoustic waves with a phase velocity of $c_{\text {phase }}$ will be enhanced through phase matching, much more than waves of any other phase velocities, if the following condition is met:

$$
\sin \theta=\frac{c_{w}}{c_{\text {phase }}} .
$$

Therefore, a wedge and transducer pair can be used for tuned wave excitation and detection as well. Examples of applying this wave tuning method to anomaly detection 


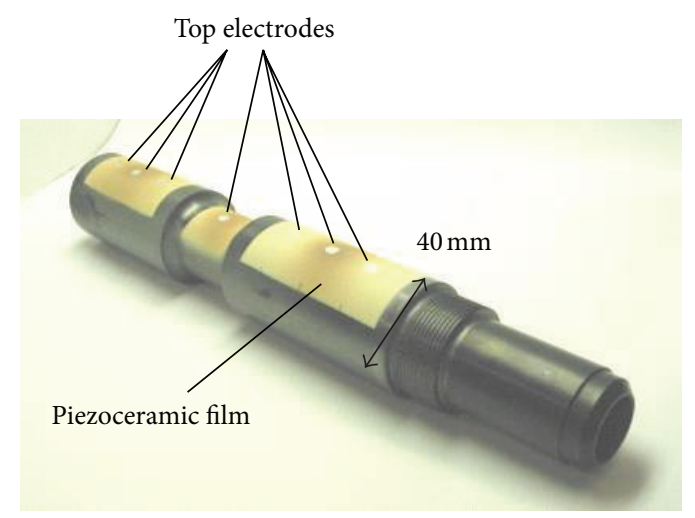

(a)

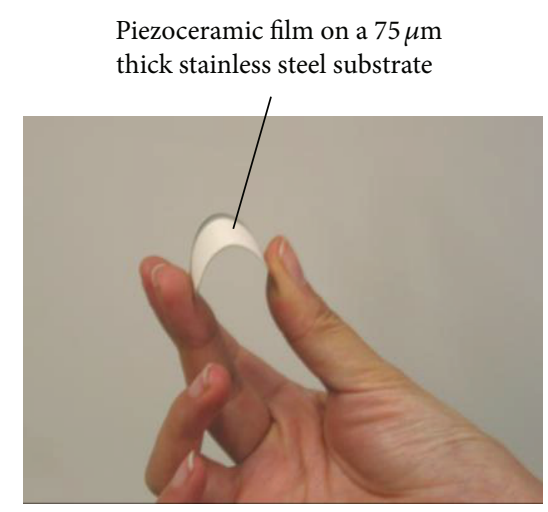

(b)

FIgURE 7: Examples of spray-on piezoelectric acoustic transducers: (a) IUTs and (b) an FUT.

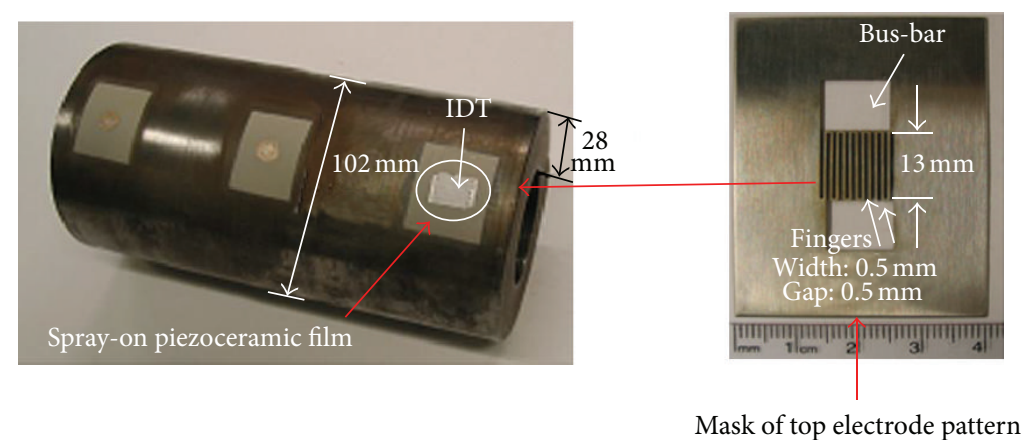

Figure 8: An interdigital transducer fabricated on a steel pipe (left) for generation and detection of Rayleigh surface acoustic waves. The interdigital top electrodes were silver-pained with help of a mask (right).

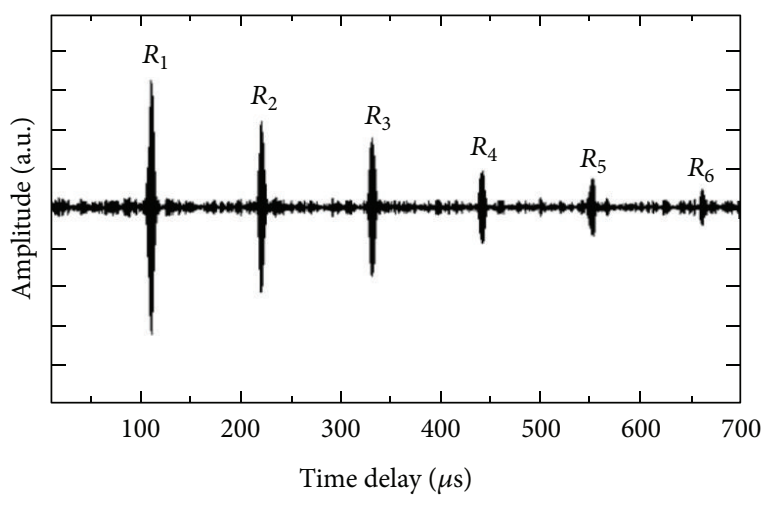

Figure 9: Rayleigh surface acoustic wave signal generated and detected with the IDT shown in Figure 8 at $150^{\circ} \mathrm{C}$.

in aircraft component can be found in [7]. Wedge based wave tuning has, however, several drawbacks. First, the phase velocity $c_{w}$ must be smaller than $c_{\text {phase }}$. This can limit either the choices of wedge materials appropriate for generation of a desired mode or the availability of modes that can be generated with a given wedge material. Secondly, spurious signals, resulting from wave reverberation inside the wedge, may deteriorate the quality of the useful signal, that is, intended applied wave. Thirdly, due to beam spreading of acoustic waves propagating through the wedge to the structure surface, other wave modes beyond the mode of interest may be generated. Furthermore, if a fixed-angle wedge is used, the efficiency of wave tuning can be affected by temperature, due to temperature dependence of wave velocities. A variable angle beam wedge can be used to cope with temperature variation or to tune wave generation to a different mode by adjusting the incident angle, as presented in Figure 4. However, in this case, an extra block introduced in the wedge is needed, which not only reduces the wave energy transferred to the structure but also creates additional wave reverberation noise.

\subsection{Tuned Wave Excitation and Detection with Piezoelectric} Wafers. Piezoelectric wafer active sensors (PWASs) made of lead zirconate titanate (PZT) and bonded onto a plate substrate have been studied theoretically and experimentally for the generation of and detection of acoustic waves. Compared with conventional ultrasound transducers, which generate and detect out-of-plane particle displacement at the transducer/structure interface, the PWAS is able to generate and detect both out-of-plane and in-plane particle displacements through thickness-wise piezoelectric coupling $\left(d_{33}\right)$ and lateral piezoelectric coupling $\left(d_{31}\right)$. If the wafer thickness 


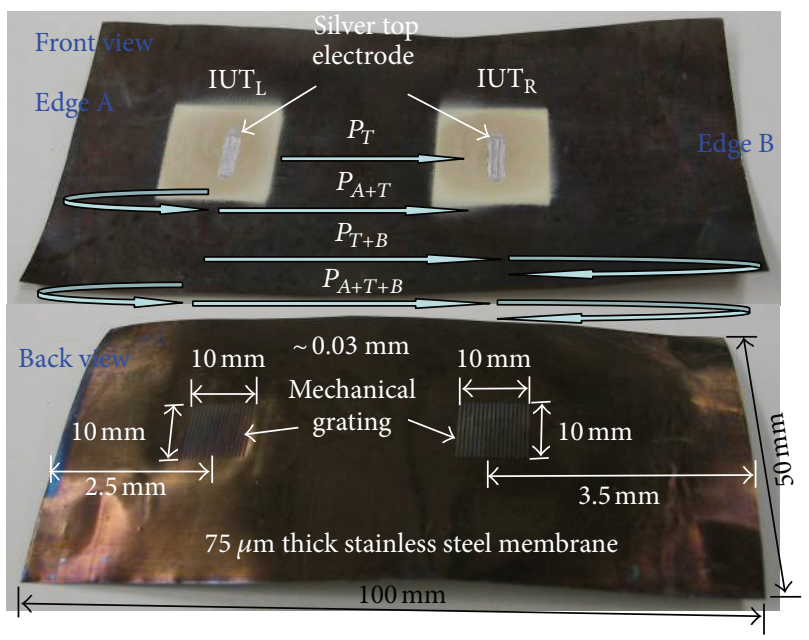

FIgURE 10: Two $62 \mu \mathrm{m}$ thick piezoceramic films are sprayed onto a $75-\mu \mathrm{m}$ thick stainless steel (SS) membrane to form two IUTs (upper pane). On the other side of the membrane and opposing each IUT, a grooved grating is made with electrical discharge machining (EDM) (lower pane). Each grating is composed of seventeen $0.3 \mathrm{~mm}$ wide, $\sim 0.03 \mathrm{~mm}$ deep, $10 \mathrm{~mm}$ long, and $0.6 \mathrm{~mm}$ equidistant grooves.

is much smaller than its lateral dimensions, the fundamental thickness-wise resonance frequency will be much higher than lateral resonant frequencies to an order equivalent to the ratio of lateral dimensions to the thickness of the piezoelectric wafer. Wave generation and detection are much more efficient when the PWAS is excited at one of its natural resonant frequencies. It happens that the most exploitable Lamb wave modes, in terms of easier excitation and detection, are the $S_{0}$ and $A_{0}$ modes in the frequency range of hundreds of $\mathrm{kHz}$. Also, in this frequency range, the coexistence and potential interference of propagating multiple higher order modes are avoided, resulting in simplified wave propagation, detection, and interpretation (of the corresponding sensor signals). Waves in this frequency range can be fairly easily generated and detected by PWASs of adequate sizes and PZT material and excited at or near one of their lateral resonant frequencies. In this configuration, the $d_{31}$ piezoelectric coupling is essential for an efficient generation and detection of $S_{0}$ and $A_{0}$ Lamb wave modes in a plate. Thickness-wise piezoelectric coupling is far less efficient for this purpose due to much higher thickness-wise resonant frequencies and also due to the fact that by using a planar $d_{31}$ effect, a more uniform direction-wise excitation and sensing capability are obtained.

It has been shown that the mode of wave motion inside a plate structure can be tuned by properly selecting the dimensions of the wafer and the excitation signal frequency and by taking into consideration the plate thickness and constituent material [8]. For example, if a square PWAS is used, efficient excitation of a particular mode of Lamb wave may be achieved if the length of the PWAS is half the wavelength of that wave at the excitation frequency. However, this excitation could still not be optimal, because this excitation frequency may not match a lateral resonant frequency of the PWAS due to different wave speeds in the wafer and in the substrate being inspected. This means that further frequency tuning (and more careful selection of PWAS material and dimensions) would be needed to achieve optimal excitation efficiency. The excitation efficiency is also affected by the bonding quality and the ratio of the stiffness of PWAS to that of the substrate at the excitation frequency. Maximum energy is transferred from PWAS to the structure and vice versa when the stiffness of PWAS matches that of the substrate [9]. Since the stiffness of a plate is proportional to its thickness, both of the thickness of PWAS and that of the substrate affect the efficiency of energy transfer. To enhance the generation of a specific mode even further, two identical PWASs may be bonded vis-à-vis onto the upper and lower surfaces of the substrate. If they are excited electrically in phase, symmetric modes will be enhanced. If the two PWASs are excited electrically out of phase, anti-symmetric modes will be enhanced.

It is important to point out that efficient excitation of a wave mode does not guarantee effective detection of this mode, since this propagating wave mode may interfere and merge with other propagating modes that coexist in the structure, or it may be so dispersive in the excitation frequency range that the wave pattern arrived at the detector has largely widened, resulting in reduced spatial and time resolutions. To achieve optimal detection ability of a desired mode, it is important to follow the four guidelines set forth in Section 2.1. The detection ability may be further improved by carefully selecting the excitation frequency and the lateral dimensions of the transducer in such a way that favors excitation of a desired mode while suppressing the generation of undesired modes. For instance, when a square wafer is used, it may be possible to select an excitation frequency at which the length of the wafer is an odd multiple of the half wavelength of a desired mode and at the same time a multiple of the wavelength of an undesired mode [10].

\subsection{Tuned Wave Excitation and Detection with Transducer} Arrays. One other way of selectively generating and detecting a desired guided wave mode is to use an array of ultrasonic transducers. In Figure 5 an example of the application of a periodic array structure of piezoelectric polyvinylidene fluoride (PVDF) transducers and an array of piezoceramic transducers is presented [3]. As in the case of a square PWAS, under uniform loading and application/installation conditions (i.e., excitation and bonding), a wave mode is most efficiently excited when the array element width is as close as possible to $1 / 2$ of the wavelength $\lambda$ of the guided wave propagating in the structure. The wave generation efficiency is further enhanced via promoting constructive interference of waves generated by all the elements when the array element spacing $d$ is equal to $\lambda$ for the excitation of waves propagating in the direction of the array. As pointed out by the authors in [3], the PVDF array has the merits of being thin, flexible, and thus convenient for conformal mounting or embedded applications. The cost of PVDF transducers is usually much lower than that of PZT transducers. On the downside, PVDF has much weaker piezoelectric strength than PZT and its lowest operation temperature (about $-30^{\circ} \mathrm{C}$ ) may be too high 


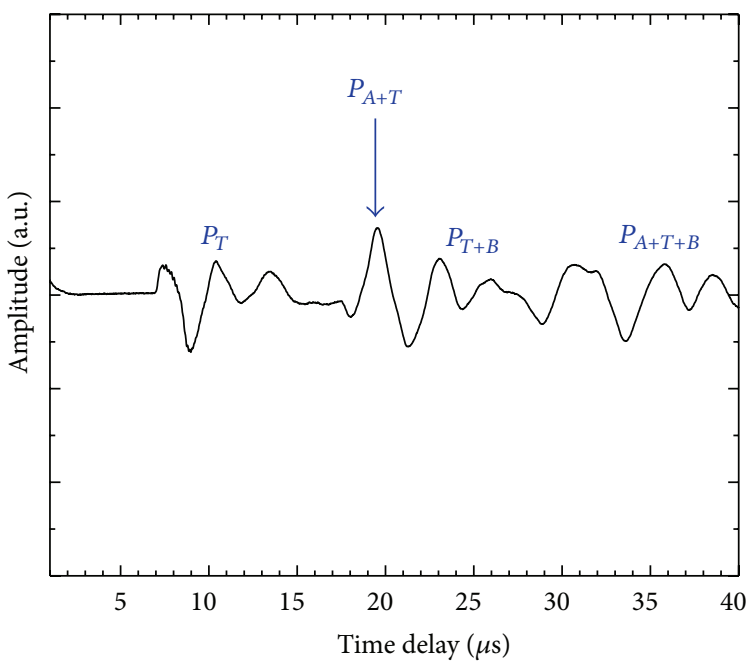

(a)

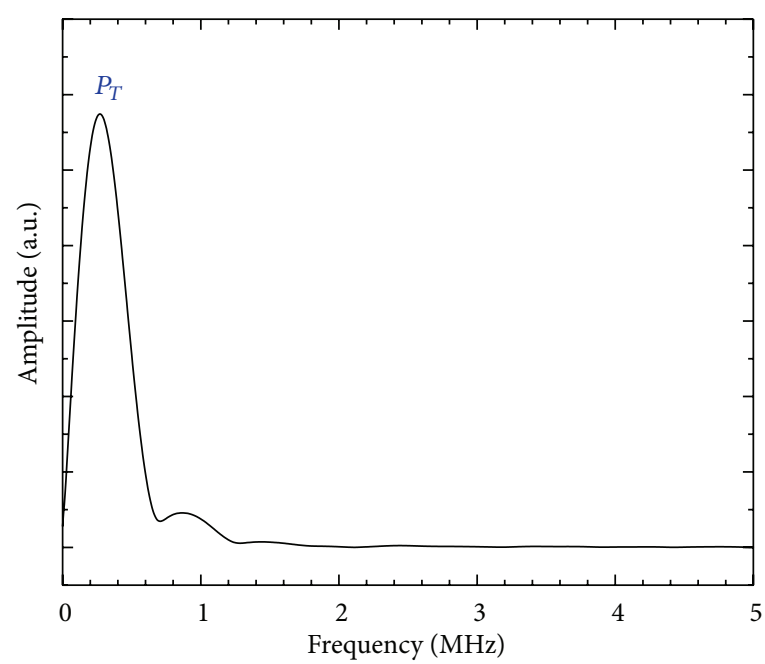

(b)

FIGURE 11: Ultrasonic performance of the IUTs shown in Figure 10 and operated in transmission mode. A 10-groove grating area was covered by the silver top electrode. (a) Signal in time domain and (b) spectrum of echo signal $P_{T}$.

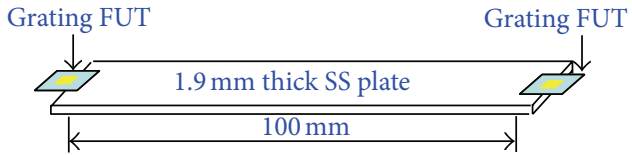

(a)

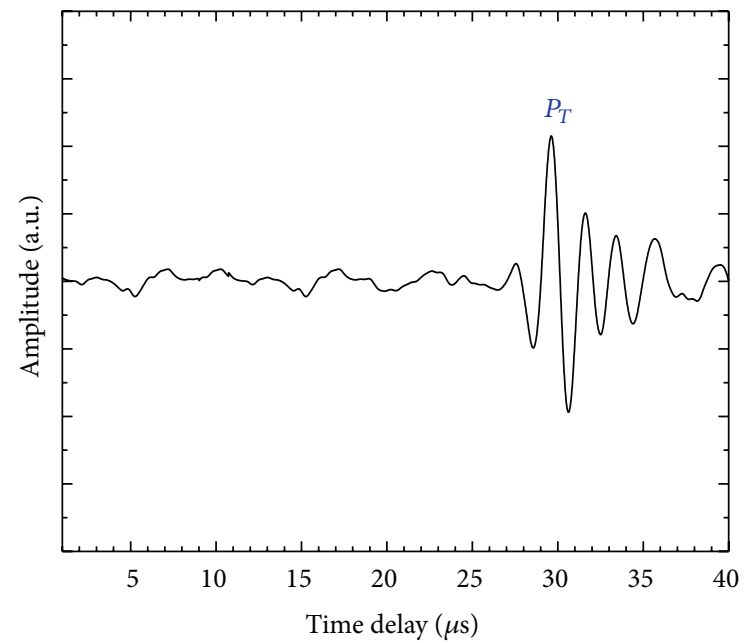

(b)

Figure 12: (a) Flexible grating transducers shown in Figure 10 are bonded at two ends of a $1.9 \mathrm{~mm}$ thick stainless steel plate. (b) Ultrasonic performance obtained by operating the two grating transducers in transmission mode.

for an application to aircraft SHM. It is important to point out that the piezoceramic transducer array in the figure was designed for pipeline monitoring, where the transducer array was pressed onto the outer surface of the pipe to realize better contact between transducers and the concerned structure.

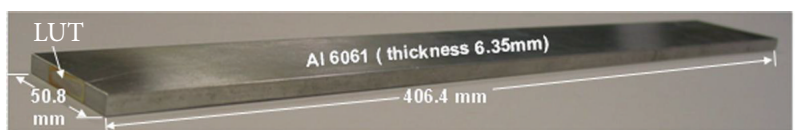

FIGURE 13: Integrated ultrasound transducer deposited onto one end edge of an aluminum plate, for generation and detection of symmetrical plate acoustic waves (PAW).

For aircraft SHM, one can bond onto aircraft structures an array of low profile piezoceramic transducers.

2.5. Tuned Wave Excitation and Detection with Phased Arrays. Wave mode tuning can be achieved by using a tuned wave phased array [12]. The array elements can be piezoelectric PZT, PVDF, or other types of acoustic transducers. The first step in constructing a tuned wave phased array would be to decide on the desired wave mode to apply and therefore on the type and dimensions of array elements and excitation frequency that would favor the generation and detection of the wave mode selected. In a simple tuned wave phased array, the array elements are mounted equidistantly onto the thinwall structure to inspect, in the direction of wave propagation, as shown in Figure 6. For illustration purpose only four elements are used for the transmitting and receiving arrays. For a tuned phased array configuration for the excitation of a particular wave mode at a selected frequency, the time interval $(\Delta \tau)$, or phase shift, in between the activation of consecutive elements in the array for the generation of the tuned wave mode is determined according to the frequency of the wave mode to apply, its propagation velocity, and wavelength (according to the dispersion relations explained previously). Let $d$ be the array element spacing, defined to be equal to the wavelength $(\lambda)$ of the wave mode to apply in an optimized configuration, according to the (excitation) 


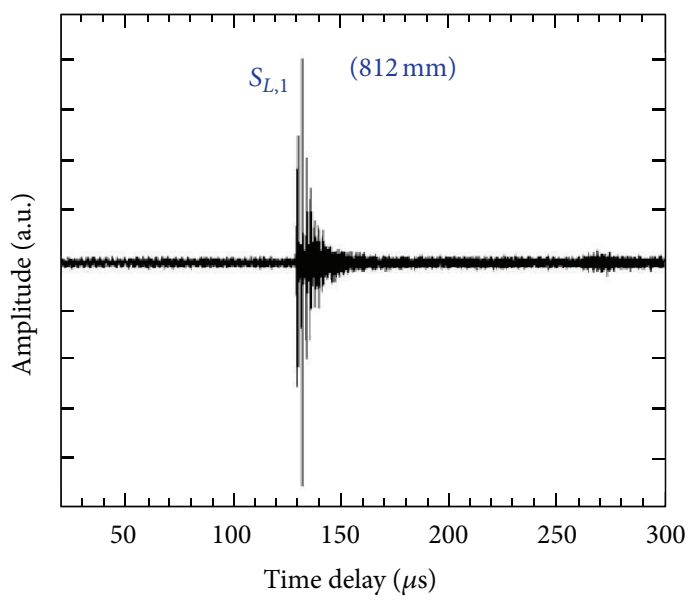

(a)

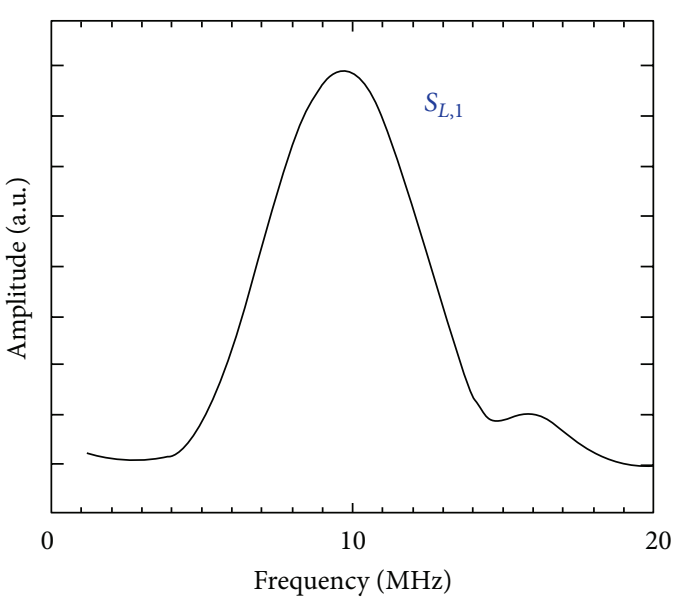

(b)

FIGURE 14: Measured reflected symmetrical PAW mode echoes from the edge opposite to the IUT (experiment performed with environment temperature of $150^{\circ} \mathrm{C}$ ) in (a) time and (b) frequency domains.

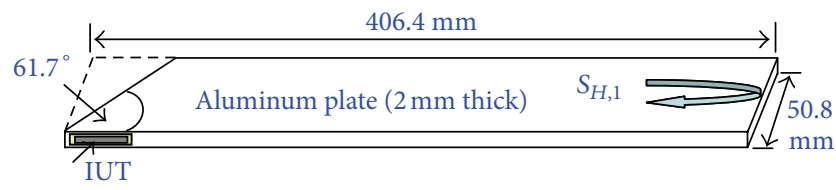

(a)

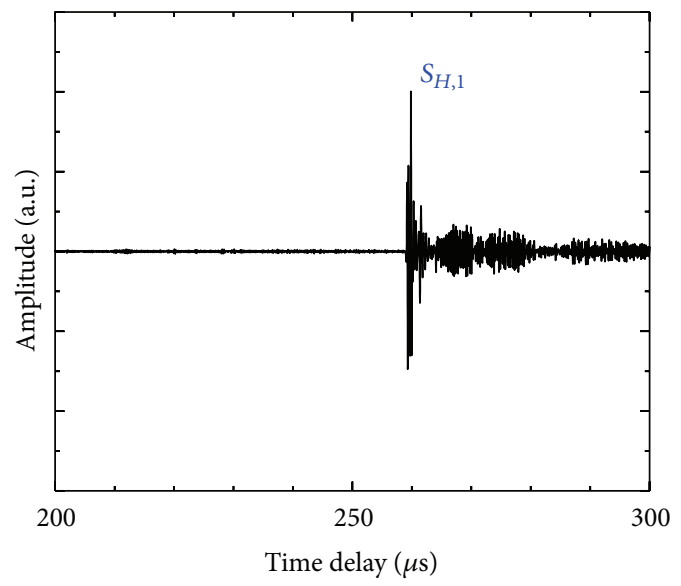

(b)

FIGURE 15: Schematic diagram of an IUT fabricated directly onto the side edge of a $2 \mathrm{~mm}$ thick aluminum plate to generate and detect $\mathrm{SH}$ PAWs (a) and a received echo signal (b).

frequency selected $(f)$ and the phase velocity $\left(c_{\text {phase }}\right)$ of a selected mode in the structure, $\Delta \tau$ is determined as the time of flight for the wave front of the selected mode to travel a distance $d$ in the structure

$$
\Delta \tau=\frac{d}{c_{\text {phase }}}\left(=\frac{\lambda}{c_{\text {phase }}}=\frac{1}{f}\right) .
$$

Array elements 1, 2, 3, and 4 are excited sequentially with excitation pulses to elements 2,3 , and 4 being delayed by $\Delta \tau$,

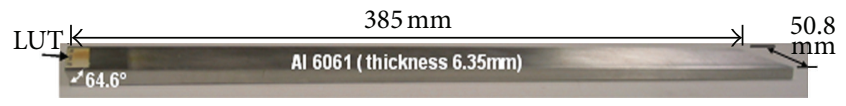

FIGURE 16: Integrated longitudinal wave ultrasound transducer (LUT) for the generation and detection of antisymmetrical PAWs using mode conversion.

$2 \Delta \tau$, and $3 \Delta \tau$ respectively with respect to the excitation pulse applied to element 1 . This ensures constructive interference of waves excited at all the elements, with these waves travelling towards the receiving array with a phase velocity $c_{\text {phase }}$. At the receiving array, the waves will be sensed and the corresponding sensor signal will be given by elements $5,6,7$, and 8 . The signals given by sensors 5,6 , and 7 will be delayed by $3 \Delta \tau, 2 \Delta \tau$, and $\Delta \tau$, respectively, with respect to the time at which the propagating wave was sensed by element 5 and then added to the signal received by element 8 . This enables the constructive interference of the signals of the different sensing elements and hence further enhances the sensitivity of the array to the propagating selected wave mode. The most significant advantage of phased array wave tuning is the capability of tuning to any desired mode (departing, however, from an optimized array since the sensor dimensions may not be optimized for inspection at the selected mode and frequency) by adjusting time delays and excitation frequency without changing the layout of array elements. This makes automatic tuning possible to compensate for temperature variation or to cope with situations where precise velocity dispersion curves are not available. The downside is the need for more sophisticated electronics than other wave tuning methods.

For selective directional generation and detection of acoustic waves, array elements can be arranged in a line, a circle, or forming a two-dimensional pattern to achieve a desired directivity pattern [15]. For example, an embedded 


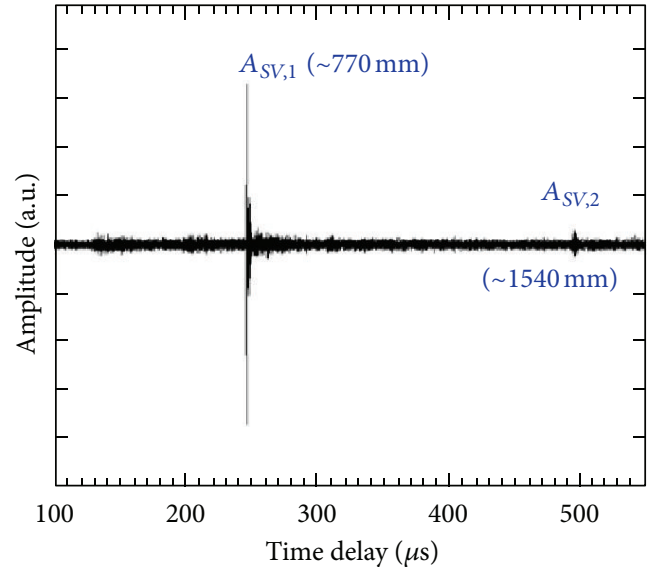

(a)

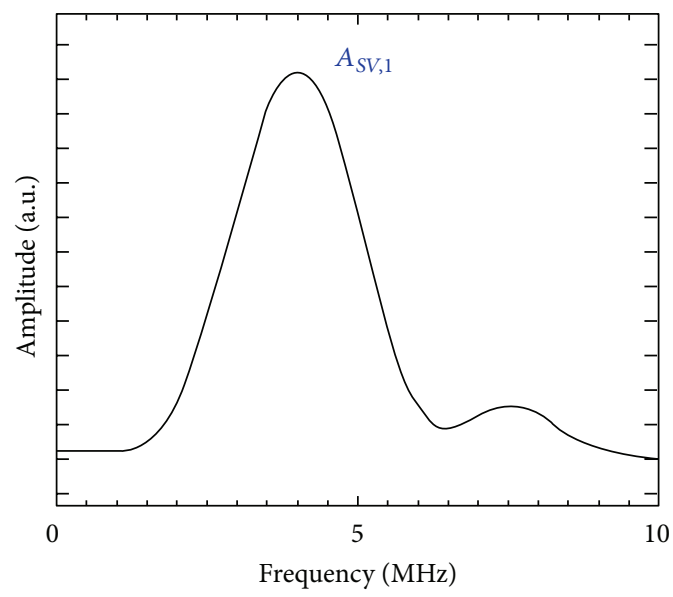

(b)

FIGURE 17: Measured anti-symmetrical PAWs reflected from the edge opposite to the IUT (experiment performed at $150^{\circ} \mathrm{C}$ ). (a) Time domain signal and (b) frequency spectrum of the 1st round trip echo signal.

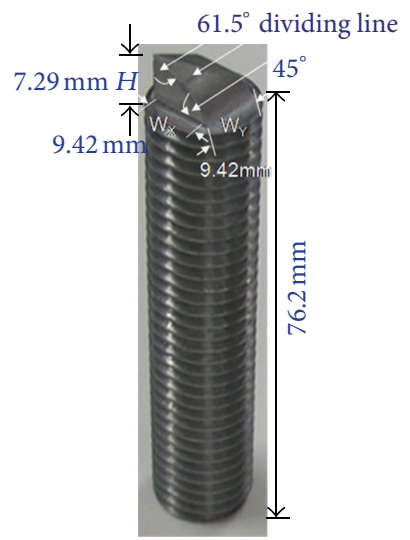

(a)

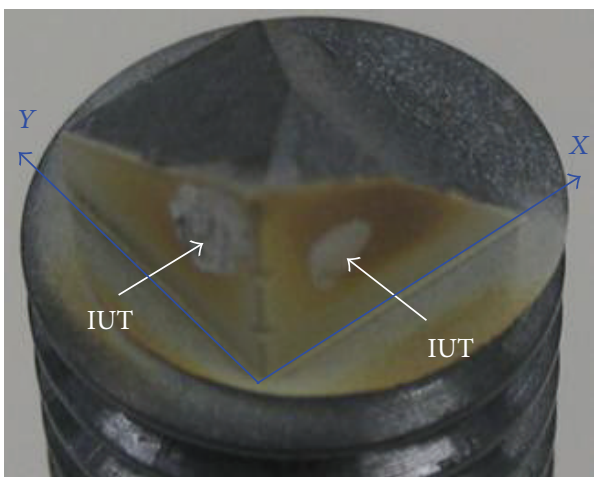

(b)

FIgURE 18: (a) An integrated probe which can generate and receive longitudinal and two orthogonal shear waves. (b) Probe head having two IUTs.

phased array can be formed by using a plurality of PWASs to scan a planar thin-wall structure in a similar way to conventional ultrasonic phased array system [16], using in the former case guided acoustic waves. By using a phased array, guided acoustic waves can also be focused to a point in a nonplanar structure, for instance, a pipe, resulting in an improved flaw detectability [17].

2.6. Wave Excitation and Detection with Sol-Gel Based Piezoceramic Transducers. A sol-gel based process has been applied to the fabrication of piezoelectric acoustic transducers $[18,19]$. A key feature in this process is the spray of a piezoceramic powder solution directly onto a substrate on which to make the transducer. This means that piezoelectric transducers could be conveniently fabricated on curved surfaces as integrated ultrasound transducers (IUTs). The process also allows fabricating flexible transducers by spraying the piezoceramic powder solution onto a thin metallic foil. The flexible ultrasound transducers (FUTs) can then be bonded onto a structure to be evaluated. An array can be formed by depositing a plurality of top electrodes directly on the piezoceramic film. Figure 7 shows two examples. Depending on the piezoceramic power used, the transducer can work at temperatures up to $200^{\circ} \mathrm{C}$ or up to $500^{\circ} \mathrm{C}$. On the lower temperature side, the transducers have been tested working at $-60^{\circ} \mathrm{C}$ in an environmental chamber.

On a microscopic level, what differentiates the sol-gel based spray-on piezoceramic transducers from conventional PZT transducers is the much higher porosity of the former. This porosity endows a spray-on ceramic film with a certain degree of flexibility, illustrated in Figure 7, and allows the spray-on type transducers to be conformally mounted onto a base structure and, as a result, to be able to sustain much higher external stresses than conventional PZT transducers without cracking. This porosity also provides a unique acoustic characteristic to sol-gel based piezoceramic acoustic transducers; that is, they are highly efficient wideband longitudinal 


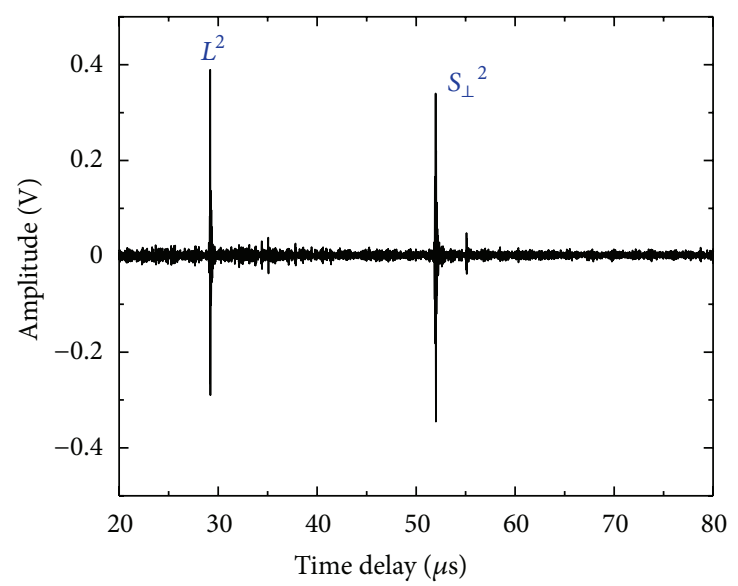

(a)

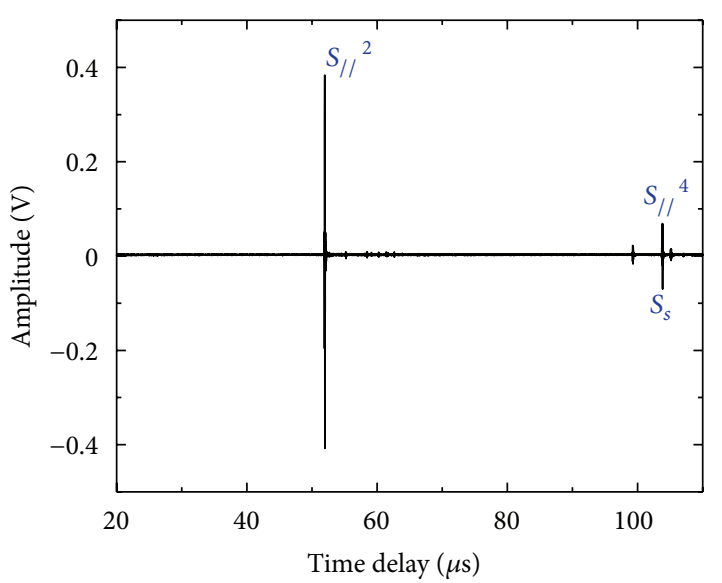

(b)

FIGURE 19: (a) Longitudinal wave echo signal $(L)$ and shear wave echo signal $\left(S_{\perp}\right)$, reflected from the probe's end. Waves generated and received by one IUT; (b) shear wave echo signal $\left(S_{\mu}\right)$, reflected from the probe's end. Waves generated and received by the other IUT. Superscripts 2 and 4 designate the first and second round trip waves, respectively.

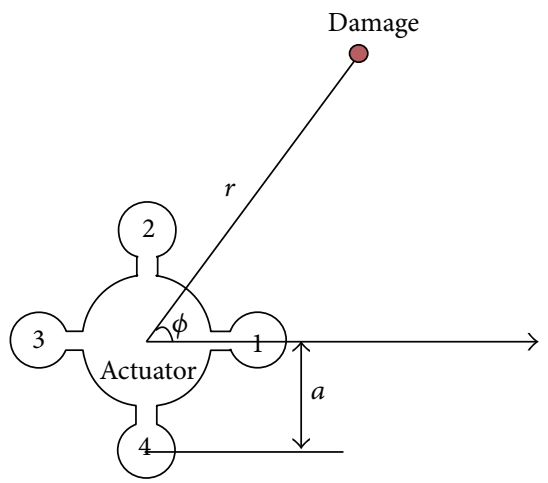

(a)

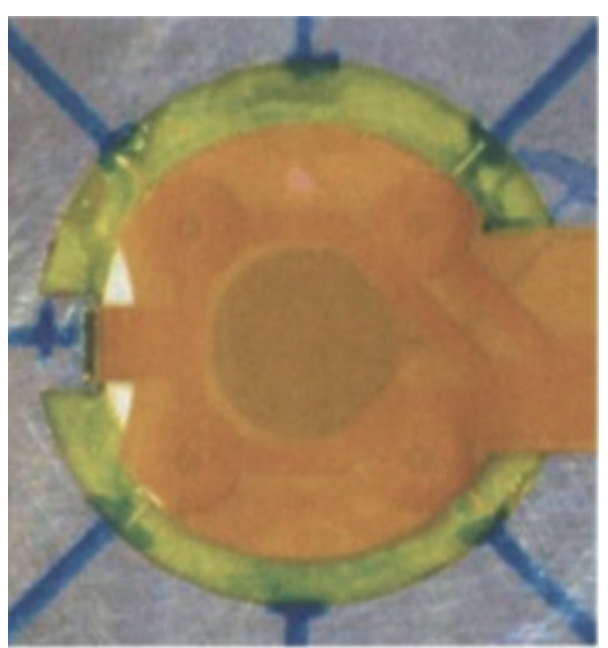

(b)

Figure 20: An SHM transducer node. (a) Schematic design; (b) packaged prototype.

wave transducers but not efficient in generating in-plane wave motions. A performance comparison study was completed between sol-gel based piezoceramic transducers and conventional PZT transducers [22]. It was found that for bulk wave generation and detection, sol-gel based transducers produce cleaner signals and generate much less crosstalk and therefore are better suited for applications where ultrasound transducer networks or bulk wave diagnostic signals with high signal-tonoise ratio (SNR) are required. Conventional PZT acoustic transducers are able to generate much stronger plate acoustic waves and therefore are better suited for applications where plate acoustic waves are used.

Sol-gel based acoustic transducers can be installed into the structures to inspect in several different ways. In Figure 8, a piezoceramic layer was first deposited onto a steel tube with $102 \mathrm{~mm}$ outer and $46 \mathrm{~mm}$ inner diameters. Then, a pair of interdigital colloidal silver electrodes were painted on the piezoceramic layer with help of a stainless steel (SS) mask to form an interdigital transducer (IDT). The width of and the gaps between the electrode fingers were designed in such a way that favored the generation and detection of Rayleigh surface acoustic waves through constructive interference, in a similar way presented in Section 2.4. In Figure 9, the sensed waves by the transducer are presented, when such waves were generated by the same transducer in an experiment with an environment temperature of $150^{\circ} \mathrm{C}$. In the figure, $R_{n}$, with $n=$ $1,2, \ldots, 6$, indicates the $n$th round trip echo signal around the cylindrical surface of the tube. The center frequency, $6 \mathrm{~dB}$ bandwidth, and SNR of the first round trip echo signal were $1.5 \mathrm{MHz}, 0.3 \mathrm{MHz}$, and $24 \mathrm{~dB}$, respectively [24].

Guided acoustic waves in a plate can also be generated by introducing line shaped mechanical gratings on the side of 


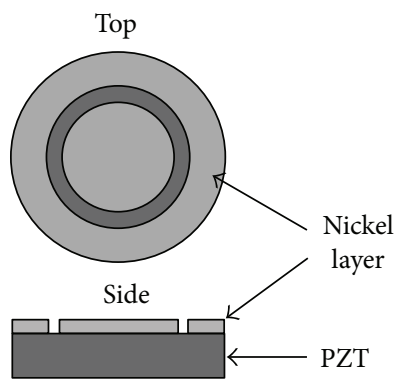

(a)

\begin{tabular}{|ll|}
\hline Excitation & Sensing \\
Dual & Dual \\
PZT A & Signal $\mathrm{Ab}$
\end{tabular}

\begin{tabular}{|cc|}
\hline Sensing & Excitation \\
Dual & Dual \\
PZT A & Signal $\mathrm{Ba}$
\end{tabular}

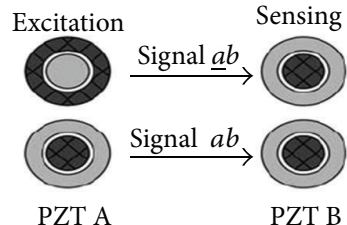

Sensing

Excitation

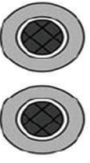

Signal $\underline{b} a$

()) Signal $b a$

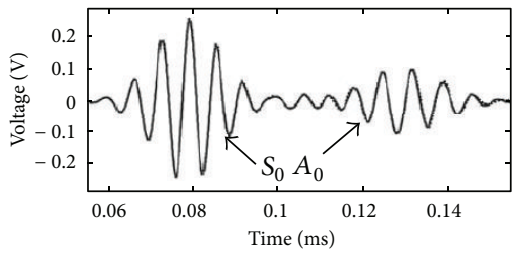

Signal $A b$

Signal $B a$

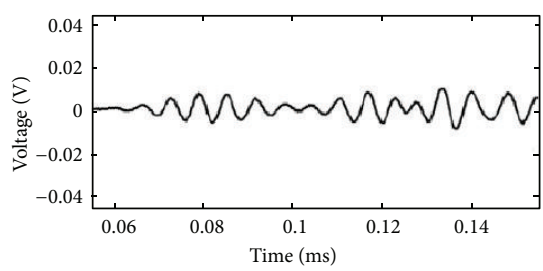

- Signal $\triangle A b$

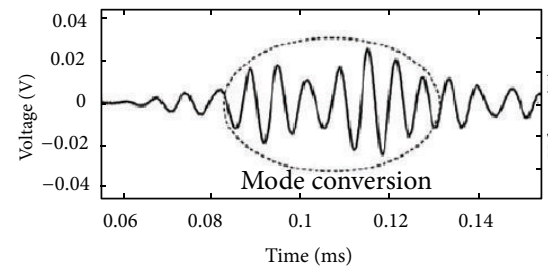

- Signal $\triangle A b$ (d)

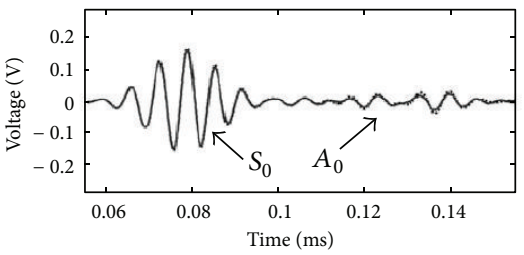

..... Signal $\underline{a} b$

— Signal $\underline{b} a$

(c)
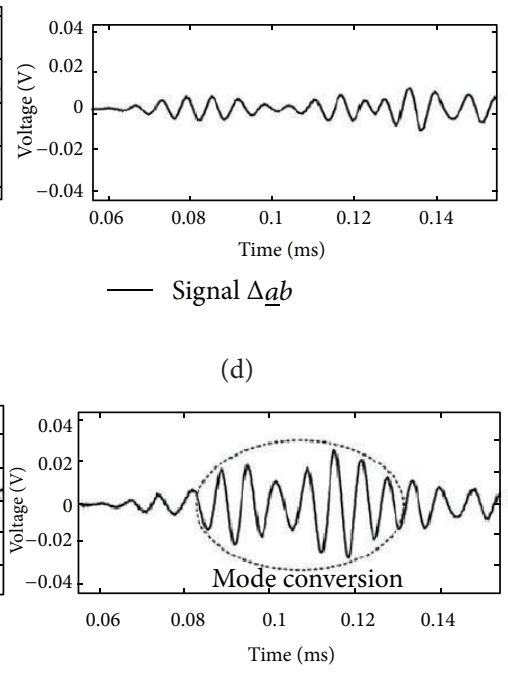

- Signal $\Delta \underline{a} b$

(e)

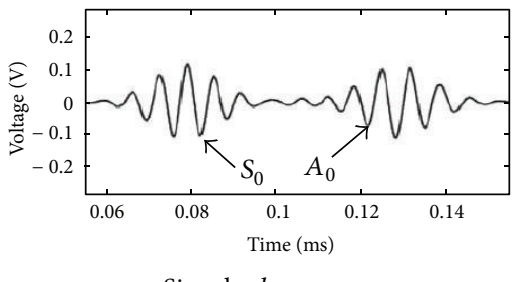

Signal $a b$

Signal $b a$

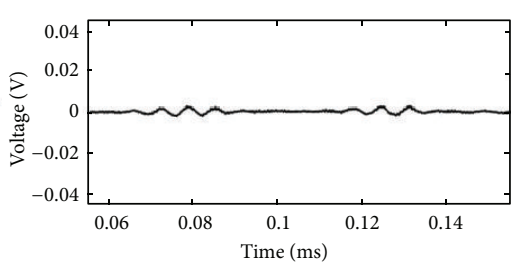

- Signal $a b$ - signal $b a$

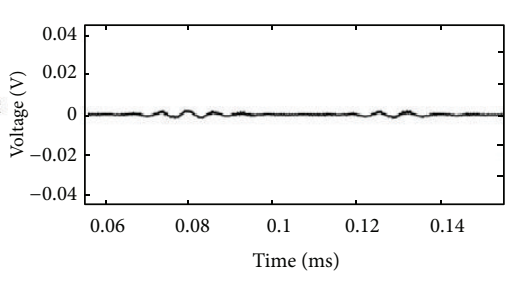

- Signal $a b-\operatorname{signal} b a$

FIGURE 21: Sensing design using dual PZT transducers. (a) A schematic drawing of a dual PZT transducer; (b) different excitation and sensing combinations; (c) signals measured with sensing configurations illustrated in (b); (d) difference between signal pairs in each of the panes of (c); (e) corresponding signal differences of those illustrated in the panes of (d) but with introduction of a notch in the plate. 


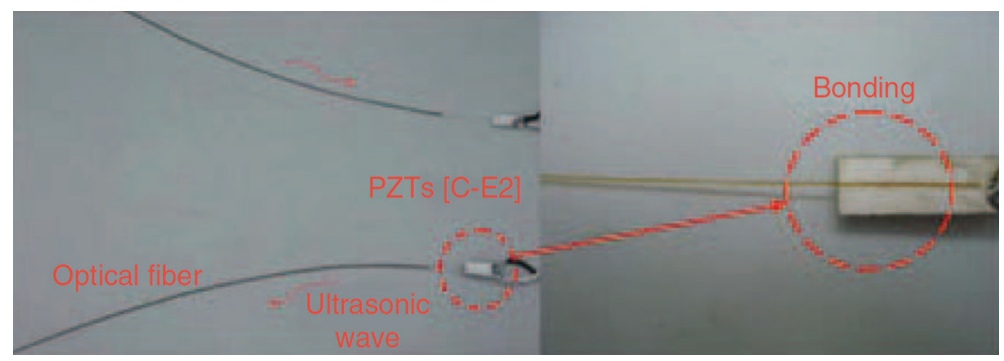

FIGURE 22: Construction of an ultrasonic active fiber sensor [11].

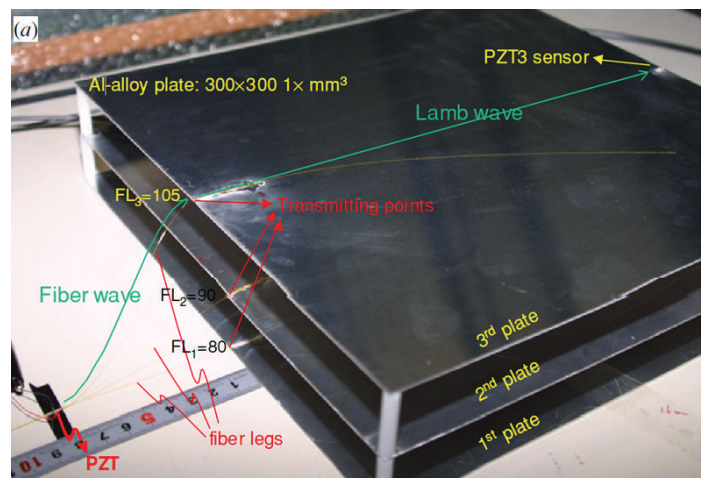

FIGURE 23: Experimental setup for ultrasonic transmission using a fiber wave PZT transducer ( FL = fiber length between the PZT in the fiber wave PZT transducer and the remote transmitting point in $\mathrm{mm}$ ). Three transmission paths, each in an individual plate, were explored simultaneously [13].
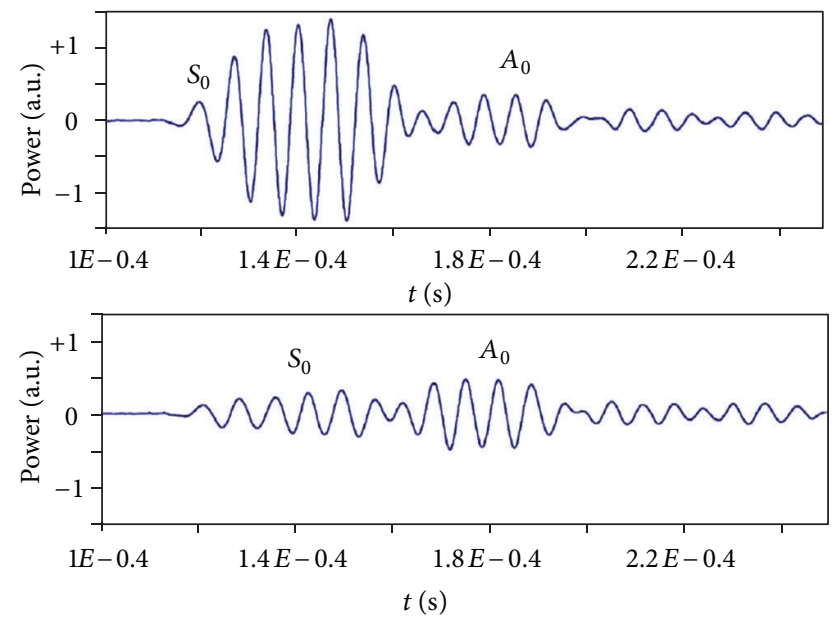

FIGURE 24: Lamb wave signals obtained by the polarimetric method for different polarizer orientations: top $-10^{\circ}$ to the plane of the plate and bottom $-60^{\circ}$ orientation [14].

the plate and exciting these gratings acoustically with a sprayon piezoceramic integrated ultrasound transducer (IUT), deposited on the opposite side of the panel with respect to the grating. This approach has been adopted for fabrication of two flexible guided wave transducers, shown in Figure 10 [26]. The width of the top electrodes is used to control the size

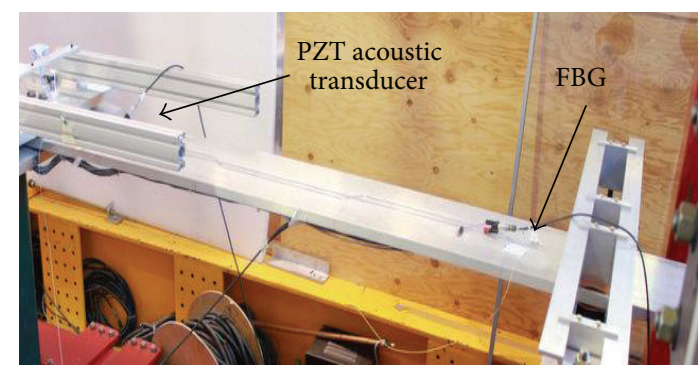

FIGURE 25: Generation and detection of acoustic waves in a $25.4 \mathrm{~mm}$ thick aluminum beam.

of active piezoelectric area. The IUTs operate predominantly in their thickness resonance mode. The gratings either convert longitudinal waves to plate acoustic waves or vice versa. In Figure 11, the signals obtained from $\mathrm{IUT}_{R}$ are presented, where sensing waves were excited by $\mathrm{IUT}_{\mathrm{L}}$. Different wave propagation paths are illustrated in Figure 10, corresponding to the different signal arrivals depicted in Figure 11. It is important to point out the difference between the $15 \mathrm{MHz}$ center frequency of the excited longitudinal waves by $\mathrm{IUT}_{\mathrm{L}}$ and the much lower center frequency of about $300 \mathrm{kHz}$ of the plate acoustic waves received at $\mathrm{IUT}_{\mathrm{R}}$.

The two grating transducers shown in Figure 10 were then separated and bonded at two ends of a $1.9 \mathrm{~mm}$ thick, $100 \mathrm{~mm}$ long, and $50 \mathrm{~mm}$ wide SS plate, as shown in Figure 12(a). The ultrasonic signal obtained in transmission mode is shown in Figure 12(b). The signal-to-noise ratio (SNR) of the received signal was $20 \mathrm{~dB}$.

In Figure 13, an IUT was fabricated onto one end edge of a $6.35 \mathrm{~mm}$ thick aluminum plate to generate and detect predominantly symmetrical Lamb wave modes. The signal reflected from the opposite end of the plate with respect to the transducer location and its frequency spectrum is shown in Figure 14 [24]. The center frequency of the signal was about $10 \mathrm{MHz}$, resulting in a frequency-thickness product value of $63.5 \mathrm{MHz} \mathrm{mm}$. By referring to Figure 1, one will see that a plurality of modes could be generated. This is indeed the case as manifested by the rather complex detected waveform.

In Figure 15, a set-up configuration to generate and detect shear horizontal plate acoustic waves (SH PAWs), with help of mode conversion, is illustrated. An IUT is deposited at the side edge near the end of a $2 \mathrm{~mm}$ thick aluminum plate with a length of $406.4 \mathrm{~mm}$ and a width of $50.8 \mathrm{~mm}$. The thickness of 


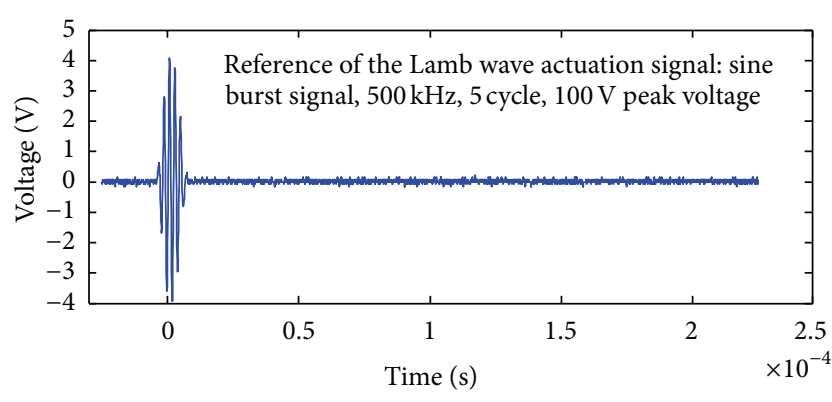

(a)

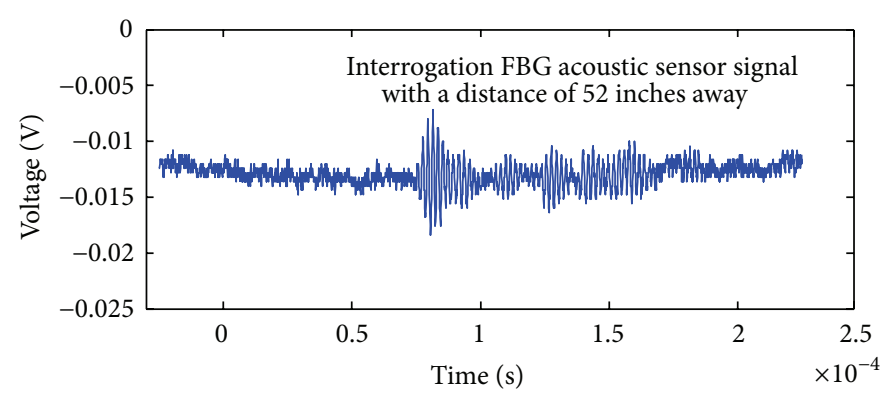

(b)

FIGURE 26: FBG response to acoustic wave excitation by a PZT transducer.

the IUT piezoceramic layer was $90 \mu \mathrm{m}$. The top rectangular electrode was $20 \mathrm{~mm}$ long and $1.6 \mathrm{~mm}$ wide, defining the IUT active area. Symmetrical PAW modes are first generated by the IUT and travel nearly $25.4 \mathrm{~mm}$ to the oblique edge, depicted in the figure, where they are converted to SH PAWs. The SH PAWs propagate then to the straight end of the plate and are reflected back to the oblique edge where they are back converted to symmetrical PAWs, which are captured by the same IUT. For this configuration the chosen mode conversion angle is $61.7^{\circ}$. This value was obtained by using Snell's law based on measured extension mode velocity and the shear wave velocity in the plate [29].

Longitudinal to shear mode conversion and vice versa can also be applied to generate and detect predominantly anti-symmetric PAWs. In Figure 16, a set-up configuration to achieve such mode conversion is demonstrated. An aluminum plate, $6.35 \mathrm{~mm}$ thick, has one of its end edge obliquely cut to form a $64.6^{\circ}$ angle between the end edge surface and the plate upper surface. This angle was calculated using Snell's law, according to the longitudinal and shear wave velocities in the plate material. At this angle, the energy conversion ratio from longitudinal waves to shear waves is $75.1 \%$, which is only $0.06 \%$ smaller than the maximum conversion ratio. Longitudinal wave ultrasound transducers, denoted by LUT in the figure, were fabricated onto the upper plate surface to its very end, above the oblique edge, by using the solgel spray technique. Acoustic waves generated by the LUT hit the oblique edge and are then mode converted to shear vertical vibrations, with the resulting boundary reflected waves propagating in a direction towards the other end of the plate. Shear vertical vibrations at the oblique edge are forced predominantly to anti-symmetric Lamb waves that propagate in the plate to its other end and then are reflected back to the oblique edge, where they are mode converted back to longitudinal waves and then captured by the LUT. In Figure 17, the signal from LUT is presented, for an experiment performed at a temperature of $150^{\circ} \mathrm{C}$. The 1 st and 2 nd round trip signals, denoted by $A_{S V, 1}$ and $A_{S V, 2}$, can be observed in the graph. The frequency spectrum of the 1st round trip echo is also shown in the figure. The $3.9 \mathrm{MHz}$ center frequency of this signal results in a frequency thickness product of 14.2 MHz-mm at which a plurality of anti-symmetrical modes are generated and propagate in co-existence in the plate [24].
Owing to the low footprint of sol-gel based acoustic transducers, it is possible to make a probe that generates longitudinal and shear waves simultaneously through mode conversion. The probe design shown in Figure 18 is able to generate a longitudinal wave and two orthogonal shear waves, simultaneously, through the use of two IUTs deposited on the probe head [31]. In Figure 19, the signals corresponding to the sensed waves (generated by the same probe and transducers) are presented. Simultaneous information of longitudinal and orthogonal shear waves' velocities can be used for the characterization of the substrate material (for example, to determine residual stresses).

2.7. Other Multielement Wave Excitation and Detection Methods. Multiple elements can be built into a single unit. Kessler and Raghavan [32], at Metis Design Corporation (MDC), patented an SHM transducer node design composed of a PZT actuator at the center and four PZT sensors equidistantly distributed in a circle, around the center actuator, as shown in Figure 20 [32]. It is claimed that through the use of this transducer node design, it is possible to determine with good accuracy a damage location, with a single node. The accuracy of this node to damage localization is limited, however, by its size.

A common SHM practice for damage detection is to compare the currently gathered data with "baseline data" previously obtained when the structure was in a pristine state or a reference condition. With the objective of detecting newer and/or developing damages, the reference condition may correspond to a structure with existing damages, which were previously detected, located, and well characterized. Strictly speaking, this approach is valid only if the performance of the sensors and associated systems remains unchanged over time. In practice, this is rarely true due to the effects of many factors on the sensors and electronics. These factors include temperature, pressure, humidity, corrosion, UV radiation, structure load, material degradation, debonding of sensors from the structure, and variation in wiring conditions. Approaches which are capable of damage identification, localization, and assessment, without the need of baseline data, and in which the effects mentioned previously in the acquired data are minimized, are deemed to be more reliable and are highly 


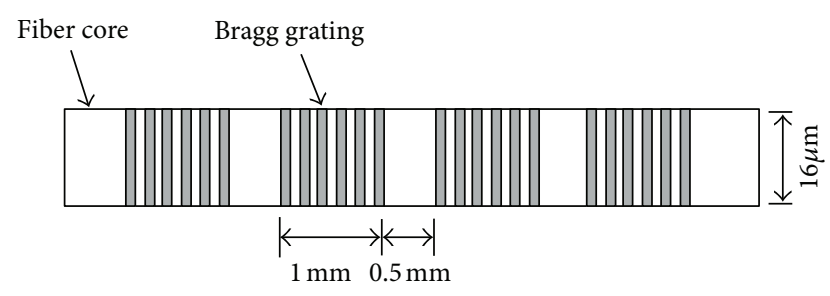

(a)

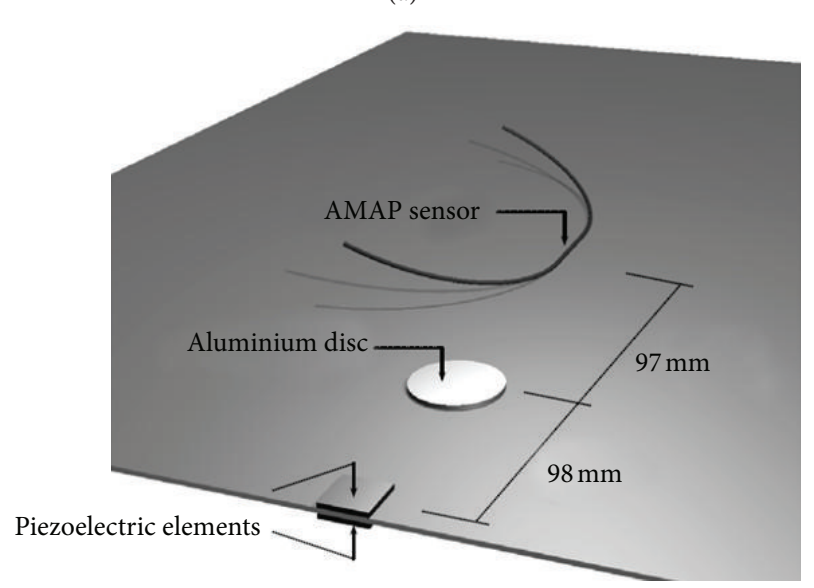

(b)
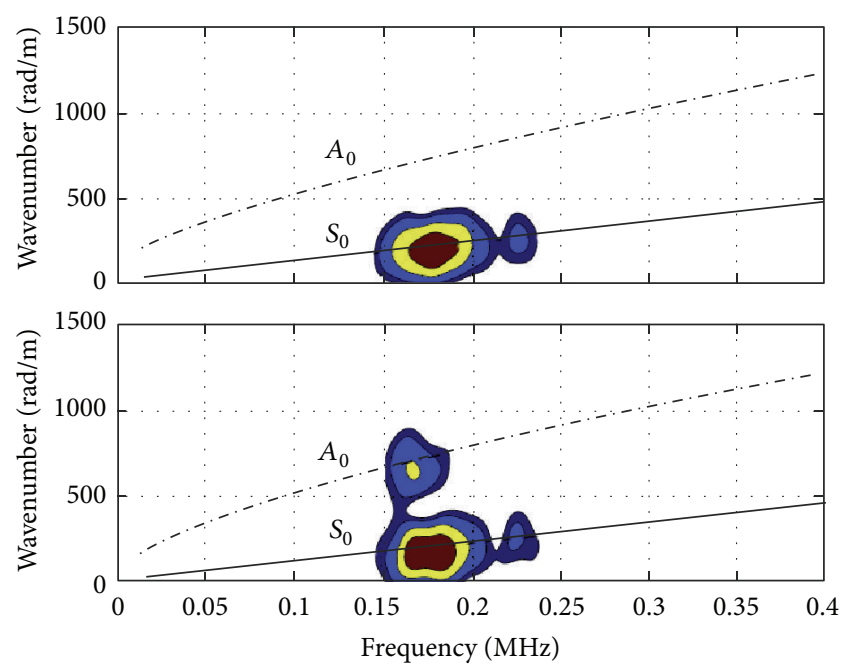

(c)

FIGURE 27: AMAP sensor demonstration. (a) Key dimensions in a section of the optical fiber core in the AMAP sensor; (b) test panel made of an aluminum alloy with the wave generation element, AMAP sensor, and bonded disc, attached to simulate a damage, as a source of reflected waves; (c) spectral decomposition of the AMAP sensor response measured for a selectively excited $S_{0}$ mode in the absence of the bonded disc (top) and after attachment of the disc (bottom) [20].

sought after. A dual PZT transducer sensing design has been proposed as a response to the need for reference-free crack detection methodologies [33]. In Figure 21(a), a schematic drawing of a dual PZT transducer is presented. One pair of such transducers is used according to six different excitation and sensing combinations, with respect to the use of the outer ring of the transducers (configuration denoted by $a$ and $b$, respectively, for the first and second transducer), the inner disc of the transducers (denoted by $\underline{a}$ and $\underline{b}$ ), and by the use of both of the inner and outer regions of the transducers simultaneously (configuration denoted by $A$ and $B$ ). These six excitation and sensing configurations are depicted in Figure 21(b), and the signals, obtained for each configuration, are presented in Figure 21(c), for a $122 \mathrm{~cm} \times$ $122 \mathrm{~cm} \times 0.6 \mathrm{~cm}$ aluminum plate without defects. With these signals, the differences between corresponding propagation and counter-propagation signal pairs can be calculated, for example, for the signal pair $A b$ and $B a$ indicated in the upper two panes of Figure 21(b). These calculated differences are depicted in Figure 21(d). In Figure 21(e), the signal differences corresponding to the ones illustrated in the panes of Figure 21(d) are displayed for the same aluminum plate but now with an introduced $0.15 \mathrm{~cm}$ deep $\times 0.10 \mathrm{~cm}$ wide $\times$ $6 \mathrm{~cm}$ long notch. The comparison between the corresponding signals in Figure 21(d) and Figure 21(e) shows that the signal differences are noticeably larger in the presence of the notch. The larger differences are believed to result from the mode conversion from $S_{0}$ mode to $A_{0}$ mode and vice versa due to the presence of the notch. The differences of the three signal pairs are used in a damage classifier method proposed by the authors to assess the damage. It should be pointed out that the proposed method assumes no mode conversion when there is no crack in the structure, that is, for a pristine structural condition. This may not be true for composite structures or for structures with more complicated geometries, with joining elements (such as fasteners and rivets) and with different boundary conditions than the plate in which this method was investigated by the authors. However, the proposed technique still has its merit in revealing defects which may not be detectable using a conventional single PZT element actuator/sensor pair.

2.8. Ultrasonic Active Fiber Sensor (UAFS) Based Pulse-Echo Method. Ultrasonic Active Fiber Sensor (UAFS) is simply a PZT acoustic transducer bonded to one end of an optical fiber, as depicted in Figure 22. The PZT transducer excites ultrasonic waves in an optical fiber, used as a wave guide, and captures ultrasonic waves reflected from the opposite end of the optical fiber or from a location in the fiber where a mechanical load is applied $[11,34]$. This sensor has been used to locate the position of the application point of a mechanical load, detect liquid spills, monitor the cure of solder, and to measure the level of liquid in a reservoir. Research efforts were dedicated to demonstrate the applicability of the PZT coupled optical fiber, as a wave guide, to transmit, generate, and acquire Lamb waves propagating in a solid media. In particular, aluminum plates were used for such demonstration [13]. In this work, the optical fiber termination, opposed to the one attached to the PZT element working as a transducer, that is, either as actuator or sensing element, was bonded to the edge of the aluminum plate. Additionally, another PZT element was bonded to the opposite edge of the plate with respect to the location where the optical fiber termination was bonded. Subsequently, either of the 


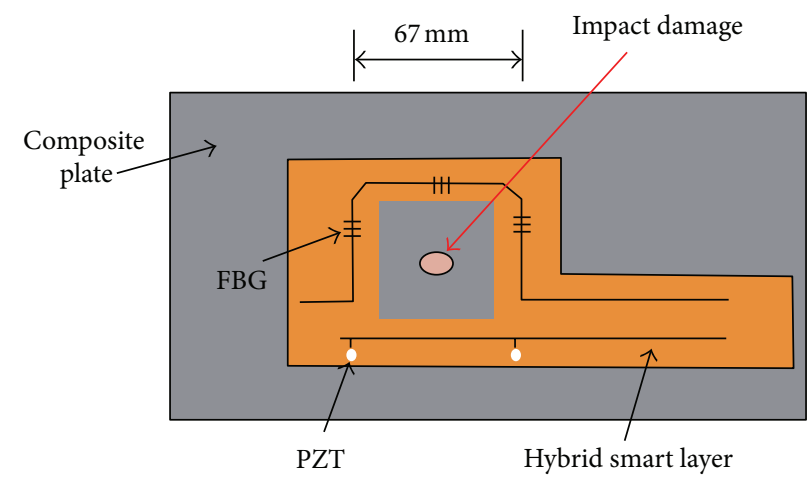

(a)

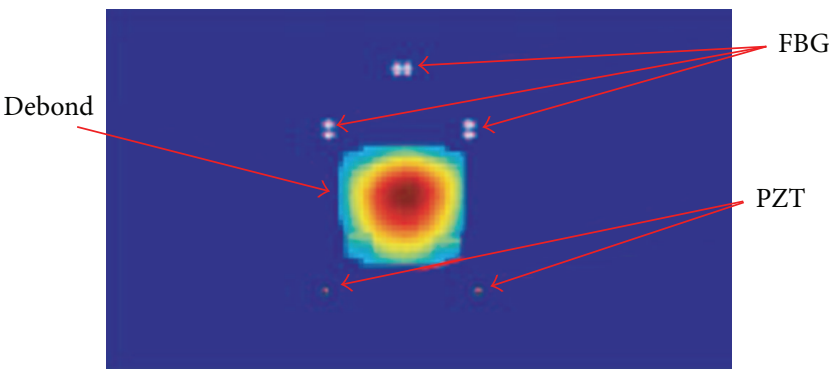

(b)

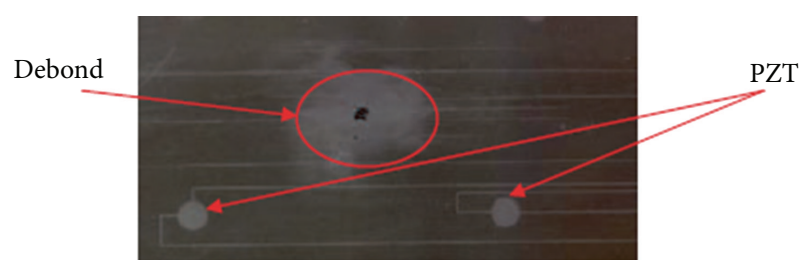

(c)

FIGURE 28: Hybrid sensor network used for damage detection. (a) Hybrid layer mounted on the surface of a composite panel; (b) diagnostic image of debond on the composite plate; (c) X-ray image of the damage [21].

PZT elements was excited while the other one was used as sensor (Figure 23). These experiments proved the capability of using the fiber wave piezoelectric transducer in a pitchcatch network configuration, with the optical fiber serving as a wave guide, to generate or sense Lamb waves propagating in the aluminum plate, with the subsequent possibility of using this transducer configuration for a damage detection system.

A main advantage of this sensor over other fiber optic sensors is its low cost, as only a common optical fiber used for telecommunications is needed, without any printed sensors. While it may not be suitable for certain embedded applications due to possibly considerable leakage of acoustic energy into the surrounding medium, this technique may be useful when embedding is not required and when the effect of bonding can be minimized or well characterized. The sensor may also be used for high temperature SHM by using the optical fiber to transfer diagnostic acoustic waves to a hot structure through a bonding layer, so that a direct contact of the PZT transducer with the hot structure is avoided. Depending on applications, the optical fiber can be replaced with wires/wave guides made of other materials.

\section{Hybrid Approaches}

3.1. Detection of Ultrasound Using Fiber Optic Sensors (FOSs). The application of Fiber Optic Sensors (FOSs) offers certain advantages in comparison with the use of conventional PZT sensors in terms of the following: directional sensitivity; more broadband response; denser sensor multiplexing; immunity to and not generating Electro-Magnetic Interference (EMI) and therefore showing good electromagnetic compatibility (EMC) with other on-board avionic systems; good mechanical and environmental durability; potential application in explosive environments, such as inside fuel tanks, possibly inside wetted structure, with minimal risk due to the fact that required sensor power and signal transmission is performed through light travelling in an optical fiber, possibly to remote locations, with the optical fibers replacing required electrical networks; and ease of embedment in composite materials without bulky wiring scheme. It has been shown that FOS can be sensitive to acoustic pressure (fiber polarimeters) or acoustic wave induced strain (using Fiber Bragg Grating (FBG) sensors) [14], and therefore, FOS can be used for detection of ultrasound. The combination of using PZT actuators for ultrasonic wave generation and FOS for diagnostic ultrasonic waves detection can be a powerful inspection tool for guided wave based SHM. Figure 24 shows that Lamb waves can be detected by using a polarimetric fiber optic sensor and that the sensitivity of the sensor to the fundamental symmetric mode $\left(S_{0}\right)$ and the fundamental anti-symmetric mode $\left(A_{0}\right)$ can be tuned by varying the polarizer orientation.

In a recent study, an FBG sensor was used to detect acoustic waves generated with a $6.35 \mathrm{~mm}$ diameter PZT acoustic transducer. The PZT transducer and the FBG sensor were 1.32 meters apart and both were bonded onto the upper surface of a $25.4 \mathrm{~mm}$ thick aluminum beam, as shown in Figure 25. The FBG sensor was able to detect acoustic waves generated by the PZT transducer at a distance of 1.32 


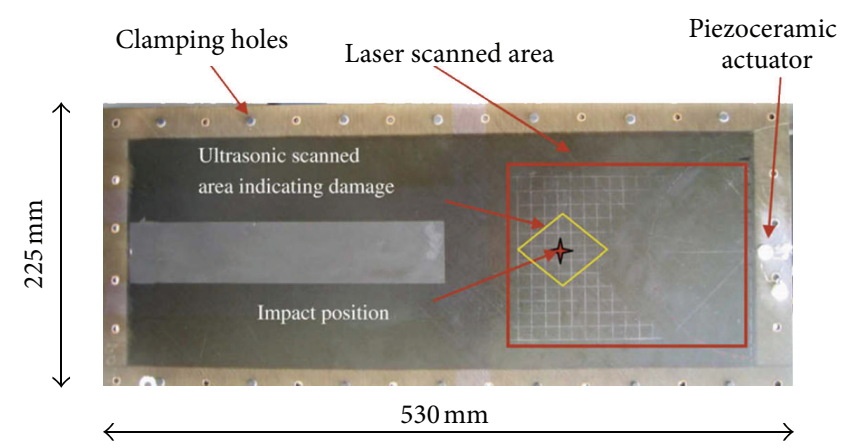

(a)

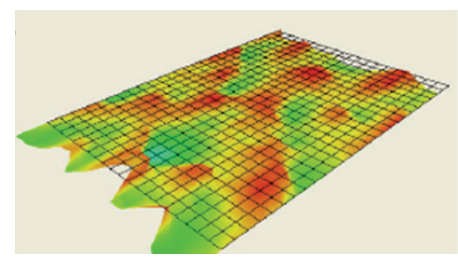

(b)

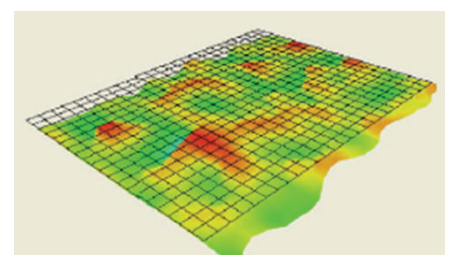

(c)

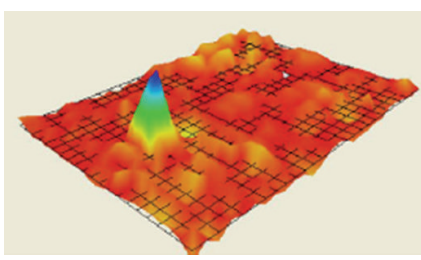

(d)

FIGURE 29: Combined use of piezoelectric actuator and laser vibrometer for SHM of aerospace composite structures. Composite plate and experimental setup used in active impact damage detection investigations (a) and Lamb wave amplitude in the in-plane $x$-direction (b), in the in-plane $y$-direction (c), and in the out-of-plane $z$-direction (d) [23].

meters from the FBG sensor, with the waves propagating in a relatively thick structural component, in comparison to the thin-wall members usually used for experimentation with guided acoustic waves. Figure 26 shows the excitation signal applied to the PZT transducer and the acoustic signal captured by the FBG sensor [35].

A distributed Bragg grating sensor array, termed acoustic mode assessment photonic (AMAP) sensor has been proposed in conjunction with the two-dimensional Fourier transform, to resolve the Lamb wave field both spatially and temporally. The method, as depicted in Figure 27, has shown its capability of the detection of structural damage by identifying acoustic wave mode conversion caused by the damage [20]. Mode conversion from $S_{0}$ to $A_{0}$ caused by the presence of an aluminum disc simulating damage, as an added discontinuity in mass and stiffness, can be clearly seen in Figure 27(c).

There are several possibilities to combine fiber optic sensors with piezoelectric actuators for guided wave based SHM. The following example shows a network configuration explored at Stanford University for damage detection in a composite laminate plate [21]. A main merit of a hybrid PZT/FBG system is the decoupling of actuator and sensor signals, whereas in an all-PZT actuating and sensing system the crosstalk between the actuation signals and sensor signals is an inherent problem particularly when highly integrated. As depicted in Figure 28, the applied system was successful in detecting, locating, and estimating the dimensions of an imposed impact resulting delamination in a composite material substrate, at the center of the sensor network. A pitch-catch based methodology was used, as well as a previously developed damage index method based on energy scattering of the waves' corresponding FBG sensor signal.
3.2. Wave Generation with Piezoelectric Actuator and Detection with Laser Vibrometer for SHM of Aerospace Composite Structures. In this method, a piezoelectric transducer is used as actuator to generate Lamb waves in a host material, such as in the carbon/epoxy composite plate depicted in Figure 29 [23]. Subsequently, a scanning 3D laser vibrometer is used to acquire Lamb wave responses from the analyzed plate and to monitor the propagation of the waves and the effect on such propagation of the interaction with existing damage. The damage introduced in the composite plate by an impact has been clearly detected by the laser vibrometer, through the out-of-plane ( $z$-direction) vibration measurement. This technique has three attractive merits. First of all, a complicated sensor layout is not required. Secondly, it is easy to apply without the need of sophisticated signal processing and wave analysis. Finally, and importantly, it does not need any baseline data as reference.

3.3. Ultrasonic Wave Generation with Laser and Wave Detection with Piezoelectric Sensor. In this technique, a laser is used for the excitation of the waves and a piezoelectric element is used as a sensor, in opposition to the technique presented in Section 3.2. The present technique uses a laser, with the help of a rotation stage, as depicted in Figure 30(a) [36], or a mirror scanner, as presented in Figure 30(b) [37], to excite, point after point, the entire surface of a structure, and uses the piezoelectric element to sense the laser induced ultrasonic waves propagating in the host material being scanned. Subsequently, all the traces of signals obtained from the piezoelectric sensor are combined to obtain a sequence of images showing the propagation of the waves in the structure, as depicted in Figure 30(c) [38]. In Figure 30(d), 


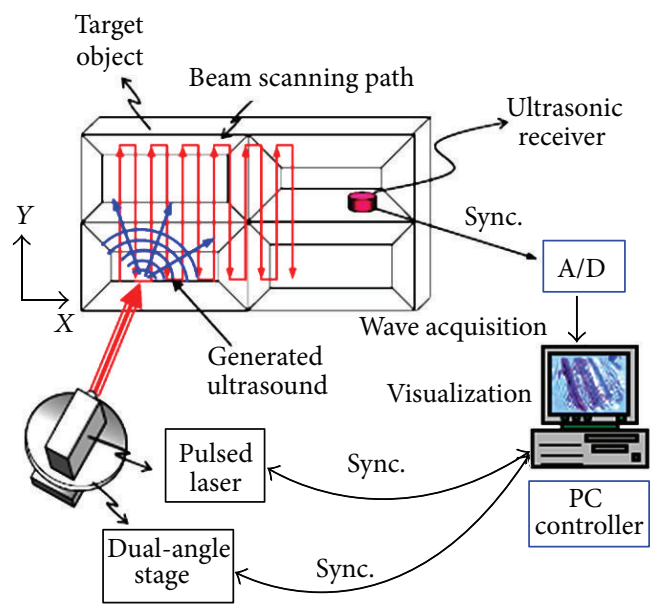

(a)

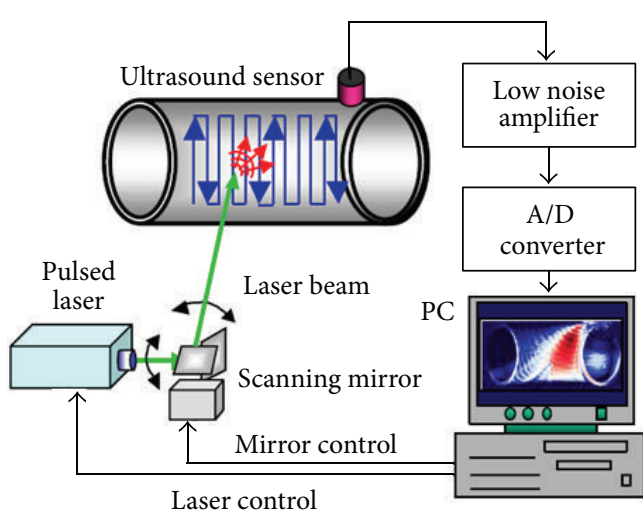

(b)

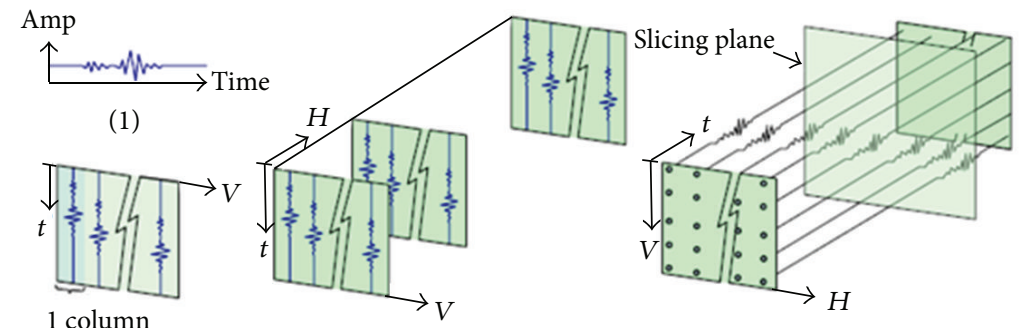

(2)

(3)

(4)

(c)
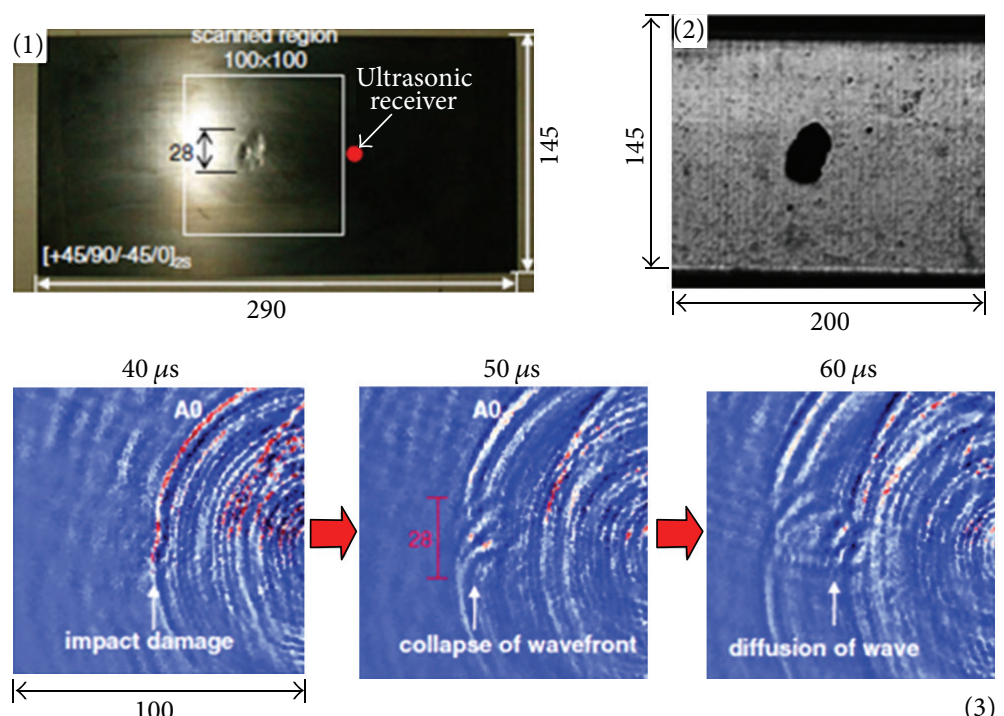

$50 \mu \mathrm{s}$

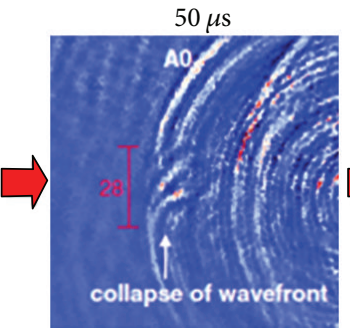

collapse of wavefront

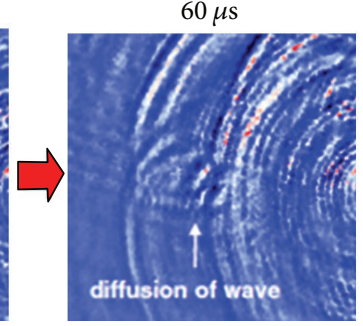

(3)

(d)

FIGURE 30: Application of a laser for ultrasonic wave generation and of a piezoelectric sensor for ultrasonic wave detection. (a) Laser ultrasonic visualization system using a dual-angle stage for scanning; (b) laser ultrasonic visualization system using a scanning mirror; (c) illustration of an image construction process: (1) one ultrasonic signal saved, (2) matrix data structure for one vertical scan, (3) raw matrix data structure for the different vertical scans, and (4) data manipulated to fit the shown structure and sliced along the time axis; (d) application example for damage detection: (1) Hammer-struck damage on a CFRP skin, (2) C-scan image, and (3) wave field propagation snapshots in the vicinity of the impact damage (dimensions in $\mathrm{mm}$ ). 


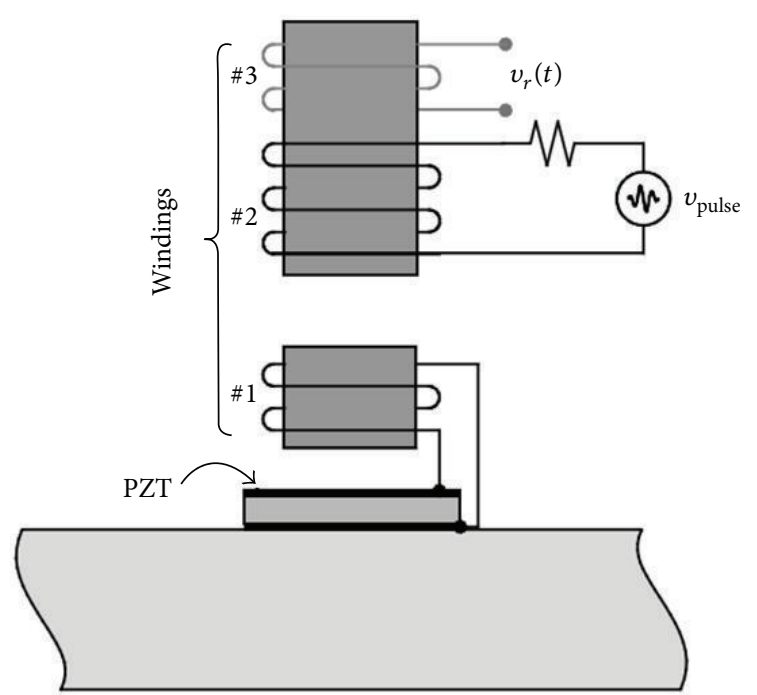

FIGURE 31: Inductively coupled PZT transducer [25].

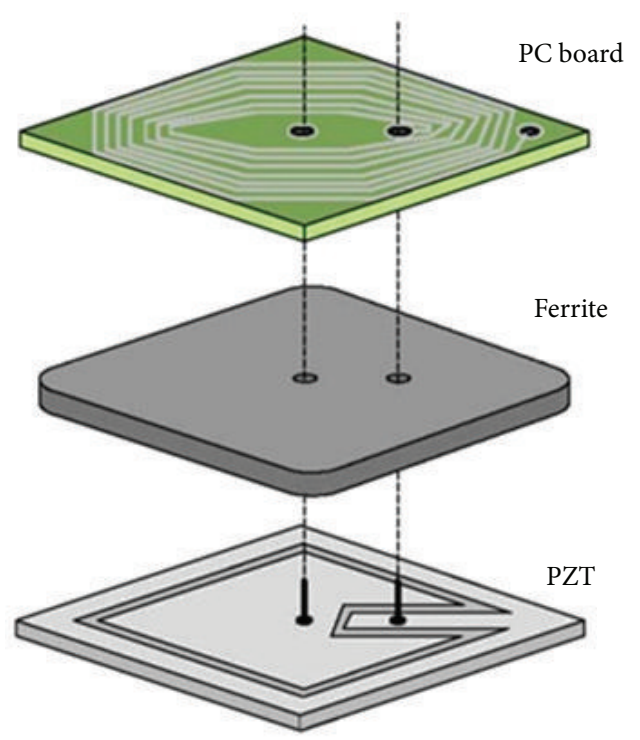

FIGURE 32: A planar transducer design using a coil on a printed circuit board [25].

an example of the application of this technique for damage detection is presented [36]. The technique has been applied to composite aircraft debonding visualization. The inspection showed that the location, shape, and size of the poor bonding defect, artificial stringer tip debonding, and real skin-spar debonding could be visualized and/or measured from the imaging results [39]. This hybrid approach has been adopted by other researchers $[40,41]$ and particularly for high temperature applications [42]. In comparison with the technique presented in Section 3.2, the current technique may present some advantages when inspecting a complex structure where it is difficult to use a piezoelectric actuator to generate detectable, strong, and diagnostic ultrasonic waves propagating through the entire structural component. By

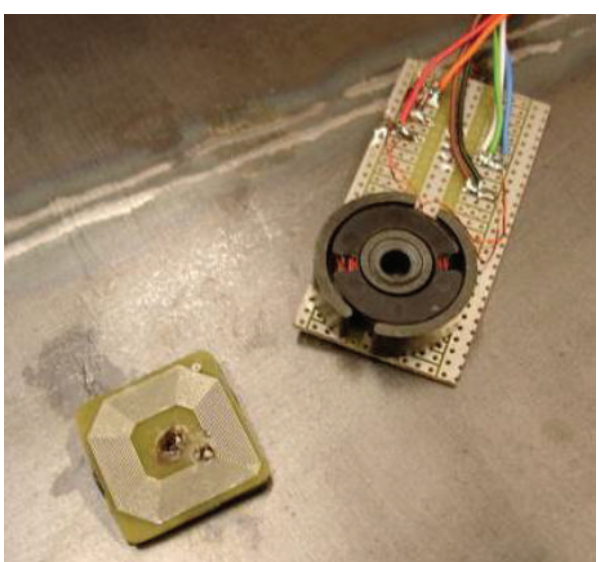

FIGURE 33: A prototype planar transducer based on the design in Figure 32 (lower left) and a pulse generation and detection probe (upper right). The PZT was $1 \mathrm{~mm}$ thick and $25 \mathrm{~mm}$ square, and the ferrite sheet was $1 \mathrm{~mm}$ in thickness and $26 \mathrm{~mm}$ square [27].

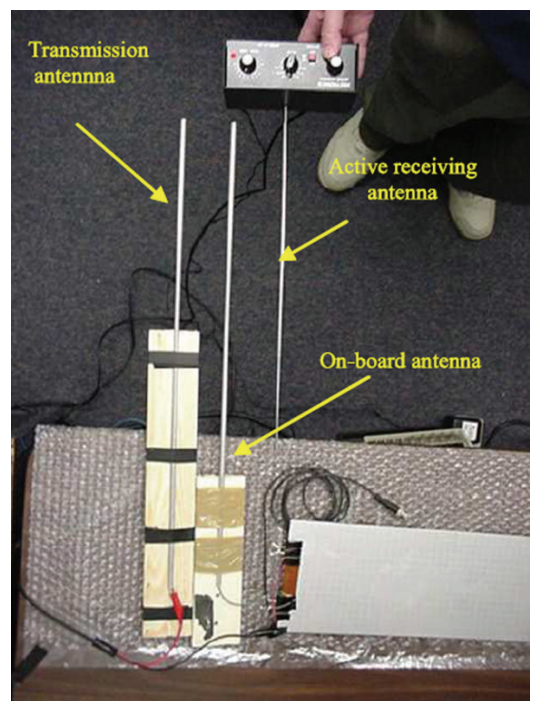

Figure 34: A wireless test setup [28].

utilizing a laser as an actuation element in the way adopted by the present technique, unlike in the selective (or tuned) mode generation of ultrasound with a piezoelectric actuator, different wave modes will unavoidably be generated in the structure. This will increase the complexity of required signal processing and data interpretation. The selection and analysis of a particular wave mode at a preferred frequency among a plurality of wave modes and frequencies in dispersive modes generated by the laser type actuation, can be tackled through wavelet transformation [43], or through analysis in the frequency-wavenumber domain [44]. One other aspect that must be taken in to account is that an ultrasonic wave generation laser usually uses powerful short laser pulses for wave generation. The applied laser beam may be detrimental to the structure, as studied by Hong et al. [45] among others. 


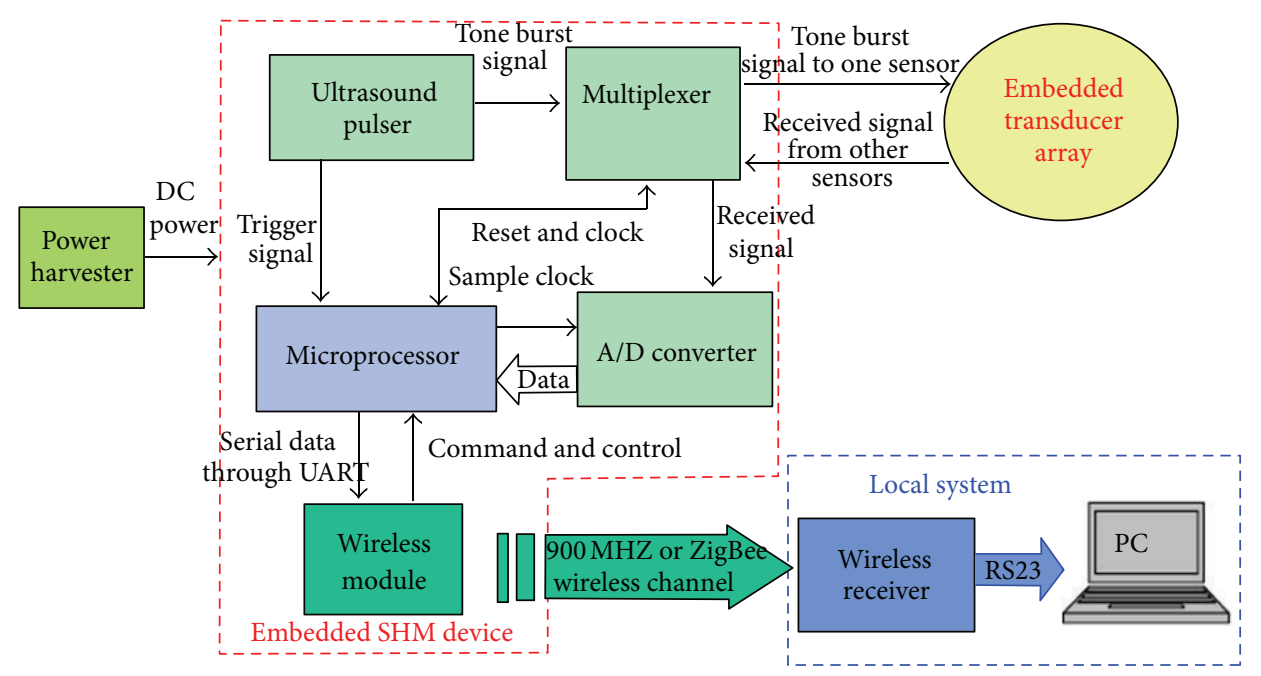

FIGURE 35: Diagram of the embedded ultrasonic structural health monitoring system [28].

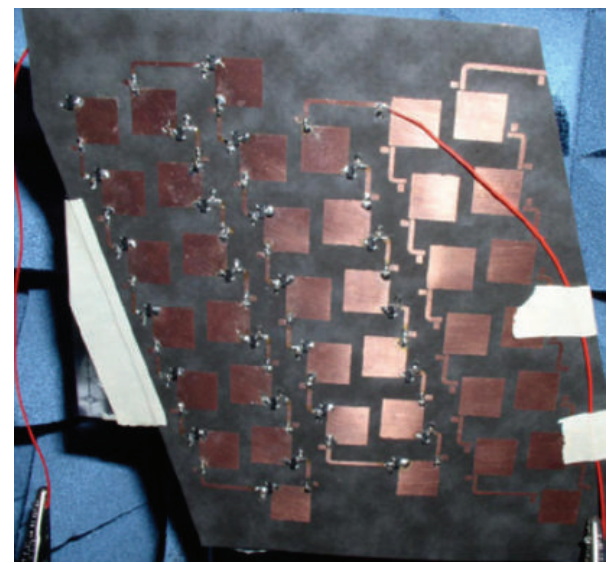

FIGURE 36: The $10 \mathrm{GHz}$ 25-element microwave rectenna array for wireless powering of on-board electronics [28].

\section{Wireless Excitation and Detection Techniques}

The present section reviews four noncontact wave generation and detection techniques that involve the use of piezoelectric acoustic transducers. These techniques aim to explore and achieve the benefits of wireless piezoelectric (mechanical) based acoustic wave actuation and sensing. These simulate the advantages presented by other noncontact transducers for acoustic wave excitation and sensing, such as electromagnetic acoustic transducers (EMATs), which base acoustic wave generation and sensing on eddy currents and electric and magnetic field conversion, transmission, and influence [46].

\subsection{Inductively Coupled Piezoelectric Acoustic Transducer.} Electrical excitation to a piezoelectric acoustic transducer can be achieved through inductive coupling. In Figure 31, this concept is depicted. A PZT wafer is wired to a winding (1) and bonded onto the substrate to inspect. The electrical excitation is sent to winding 2 and coupled to winding \#1 inductively to excite the PZT wafer. Ferrite cores are used to increase the mutual inductance. The PZT wafer, when excited electrically, generates acoustic waves in the structure through converse piezoelectric effect. The same setup can also sense acoustic waves propagating in the structure via piezoelectric effect and inductive coupling. If operated in pulse/echo mode, an additional coil, winding 3 , can be used for sensor signal output [25]. Depending on the characteristics of the probes, the separation of the excitation and sensing probes (windings 2 and 3) from the sensing element (PZT and winding 1) can be as large as $20 \mathrm{~cm}$ [47] and allows both the probe and the sensing element to be completely sealed, therefore offering a superior protection. Since the sensing element can be entirely built in a compact enclosure, it could be conveniently embedded in or mounted on a structure for structural health monitoring [25]. Figure 32 illustrates a planar transducer design using a coil on a printed circuit board. The PZT top surface is patterned to form two electrodes. Connections are made through a ferrite sheet to a printed circuit board containing a two-layer and spiral-wound coil [25]. A prototype of this design, paired with a pulse generation and detection probe with winding 2 wound on a ferrite pot core of similar size, is shown in Figure 33. This transducer pair was able to generate and detect $S_{0}$ and $A_{0}$ modes of Lamb waves in a $3.2 \mathrm{~mm}$ thick steel plate girder in a frequency range from $250 \mathrm{kHz}$ to $351 \mathrm{kHz}$ and for a probe-to-transducer coil distance up to $6 \mathrm{~mm}$. The inductively coupled non-contact wave generation and detection approach could be highly beneficial to applications for inspection of a mobile structure, for example, a helicopter rotor blade, by bonding a sensing element on the blade while keeping the excitation/sensing probe(s) on a fixed base at a close distance to the blade. A diagnostic signal can be generated and detected when the sensing element passes in front of the excitation/sensing probe(s). This configuration enables a minimization of the weight of the transducer system installed in the rotating component with a view to respect the small maximum weight 


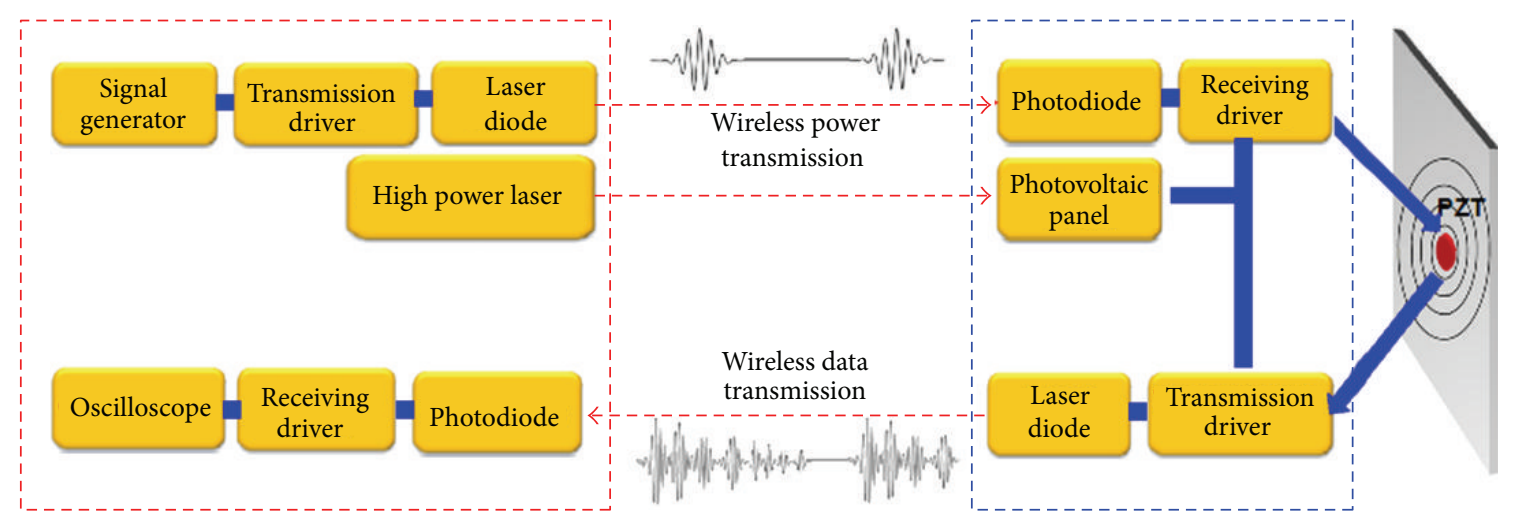

FIGURE 37: A schematic for optics based wireless guided wave generation and sensing [30].

that can be added to such rotating components (restricted by the operation conditions of such structures, in terms of their inertia, vibration, balancing, etc.).

\subsection{Capacitively Coupled Piezoelectric Acoustic Transducer.} Capacitive coupling is another approach to wireless acoustic wave excitation and detection. In Figure 34, a test setup is presented, in which a PVDF transducer is bonded to one end of an aluminum plate. A transmitting monopole antenna delivers radio frequency (RF) pulses wirelessly through an on-board monopole antenna to the PVDF transducer for generating Lamb waves, and another active monopole receives the ultrasonic echoes from the PVDF sensor through the same on-board monopole antenna. The antennas are made of $6.5 \mathrm{~mm}$ diameter aluminum tubes [28]. A fundamental limitation of this approach is the required short coupling distance $(<10 \mathrm{~cm})$. Large antenna size can also be an issue for practical applications.

\subsection{Microwave Powered Wave Generation and Detection.} Microwaves are electro-magnetic waves in the $300 \mathrm{MHz}$ to $300 \mathrm{GHz}$ frequency range. These frequencies are too high for the generation and detection of acoustic waves through direct coupling with conventional piezoelectric acoustic transducers which usually operate in the tens of $\mathrm{kHz}$ to several tens of $\mathrm{MHz}$ range for most NDE/NDI applications. However, microwave energy can be used to power an embedded piezoelectric based diagnostic device. In Figure 35, the diagram of an embedded ultrasonic SHM system is presented [28]. As embodiment, the power for the embedded sensor system was provided wirelessly from a $10 \mathrm{GHz}$ transmitter and horn antenna at a $1 \mathrm{~m}$ range from the inspected aircraft wing. A $10 \mathrm{GHz}$ patch antenna array loaded with rectifiers (rectenna array) was conformably mounted onto the inspected wing to convert incident microwave power to DC power, as depicted in Figure 36.

4.4. Wireless PZT Excitation and Sensing with Laser. Lasers have also been used to generate and transmit power wirelessly to achieve non-contact piezoelectric based acoustic wave generation and sensing. A schematic of the concept is given in Figure 37. An electric waveform generated with a signal generator is converted into a laser power modulation using a transmission $(T x)$ driver, and the laser wirelessly transmits a generated waveform to a photodiode. The photodiode converts the received laser light modulated intensity into electric current (proportionally to the received light power). The generated current signal is then converted into proportional electric voltage, through on-board electronic circuitry, by a receiving $(R x)$ driver, which is used to excite the PZT attached onto the host structure. The excited PZT consequently creates diagnostic guided waves within the structure. The reflected waves are measured by the same PZT and reconverted into a laser power modulation using the on-board laser and Tx driver. The laser light with modulated power is transmitted back to another photodiode located in the data acquisition unit for diagnosis. Again, in this photodiode the variations in the received laser light intensity (proportional to the PZT sensor signal and to the detected waves) are translated to corresponding variations in an electrical signal, which can be interpreted for wave detection analysis. An additional $5 \mathrm{~W}$ high power laser is utilized to supply the power necessary for operation of the Tx and $R x$ drivers at the PZT transducer node [30]. The laser based approach enabled the excitation of the transducer and the acquisition of diagnostic signals with the laser and transducer being separated by distances of around $0.6 \mathrm{~m}$ to $1 \mathrm{~m}$ (with the working pairs of emitting lasers and receiving photodiodes having to be in-line of sight between each other). This feature could be beneficial for inspection of areas which are difficult to access. Owing to its capability of transmitting arbitrary waveforms and capability of the PZT to sense both in-plane and out-of-plane vibrations, the technique could be better suited for tuned wave generation and detection than conventional laser ultrasonics. Examples of potential applications and usefulness for this system are presented in [48-51].

\section{Conclusions}

In this paper, different concepts and a number of approaches to generating and detecting diagnostic acoustic waves for SHM through the use of piezoelectric acoustic transducers are reviewed. All approaches aim to address three basic 
requirements to attain a successful SHM system and application: generating a diagnostic wave which is effective in revealing structure integrity information; generating and detecting the desired wave in an efficient manner; generating and detecting the desired wave in a way that is practical. Although this paper is divided into three categories, some of the concepts may be fused together to form a more powerful solution to a specific problem.

\section{References}

[1] J. L. Rose, "Dispersion curves in guided wave testing," Materials Evaluation, vol. 61, no. 1, pp. 20-22, 2003.

[2] J. L. Rose, "Waves in plates," in Ultrasonic Waves in Solid Media, pp. 101-131, Cambridge University Press, New York, NY, USA, 1999.

[3] T. R. Hay and J. L. Rose, "Flexible piezopolymer ultrasonic guided wave arrays," IEEE Transactions on Ultrasonics, Ferroelectrics, and Frequency Control, vol. 53, no. 6, pp. 1212-1217, 2006.

[4] H. Lamb, "On waves in an elastic plate," Proceedings of the Royal Society of London. Series A, vol. 93, pp. 114-128, 1917.

[5] V. Giurgiutiu, "Tuned Lamb wave excitation and detection with piezoelectric wafer active sensors for structural health monitoring," Journal of Intelligent Material Systems and Structures, vol. 16, no. 4, pp. 291-305, 2005.

[6] J. L. Rose, "Ultrasonic guided waves in structural health monitoring," Key Engineering Materials, vol. 270-273, no. I, pp. 14-21, 2004.

[7] J. L. Rose and L. E. Soley, "Ultrasonic guided waves for anomaly detection in aircraft components," Materials Evaluation, vol. 58, no. 9, pp. 1080-1086, 2000.

[8] V. Giurgiutiu, "Tuned waves generated with piezoelectric wafer active sensors," in Structural Health MonitorIng With Piezoelectric Wafer Active Sensors, pp. 309-362, Academic Press, San Diego, Calif, USA, 1 edition, 2008.

[9] V. Giurgiutiu, "Piezoelectric wafer active sensors," in Structural Health Monitoring with Piezoelectric Wafer Active Sensors, pp. 297-300, Academic Press, San Diego, Calif, USA, 1 edition, 2008.

[10] B. Rocha, Structural Health Monitoring of Aircraft Structures, Instituto Superior Tecnico (IST), Lisbon, Portugal, 2011.

[11] S. H. Lim, I. K. Oh, and J.-R. Lee, "Ultrasonic active fiber sensor based on pulse-echo method," Journal of Intelligent Material Systems and Structures, vol. 20, no. 9, pp. 1035-1043, 2009.

[12] S.-C. Wooh, “Tuned wave phased array," USA Patent 6,728,515 B1, 27, April 2004.

[13] J.-R. Lee and H. Tsuda, "Investigation of a fibre wave piezoelectric transducer," Measurement Science and Technology, vol. 17, no. 9, pp. 2414-2420, 2006.

[14] B. Culshaw, G. Thursby, D. Betz, and B. Sorazu, "The detection of ultrasound using fiber-optic sensors," IEEE Sensors Journal, vol. 8, no. 7, pp. 1360-1367, 2008.

[15] V. Giurgiutiu, "In-situ phased arrays with piezoelectric wafer active sensors," in Structural Health monitorIng With Piezoelectric Wafer Active Sensors, pp. 503-588, Academic Press, New York, NY, USA, 2008.

[16] V. Giurgiutiu and J. Bao, "Embedded-ultrasonics structural radar for in situ structural health monitoring of thin-wall structures," Structural Health Monitoring, vol. 3, no. 2, pp. 121140, 2004.
[17] J. L. Rose, Z. Sun, P. J. Mudge, and M. J. Avioli, "Guided wave flexural mode tuning and focusing for pipe testing," Materials Evaluation, vol. 61, no. 2, pp. 162-167, 2003.

[18] M. Kobayashi and C.-K. Jen, "Piezoelectric thick bismuth titanate/lead zirconate titanate composite film transducers for smart NDE of metals," Smart Materials and Structures, vol. 13, no. 4, pp. 951-956, 2004.

[19] M. Kobayashi, C.-K. Jen, J. F. Bussiere, and K.-T. Wu, "Hightemperature integrated and flexible ultrasonic transducers for nondestructive testing," NDT \& E International, vol. 42, no. 2, pp. 157-161, 2009.

[20] N. Rajic, C. Davis, and A. Thomson, "Acoustic-wave-mode separation using a distributed Bragg grating sensor," Smart Materials and Structures, vol. 18, no. 12, Article ID 125005, 2009.

[21] Z. Wu, X. P. Qing, and F. K. Chang, "Damage detection for composite laminate plates with a distributed hybrid PZT/FBG sensor network," Journal of Intelligent Material Systems and Structures, vol. 20, no. 9, pp. 1069-1077, 2009.

[22] K.-T. Wu, Z. Sun, and N. Mrad, "A comparative study on two families of integratable ultrasound transducers for structural health monitoring," in Proceedings of the International Workshop on Smart Materials \& Structures and NDT in Aerospace, 2011.

[23] W. J. Staszewski, S. Mahzan, and R. Traynor, "Health monitoring of aerospace composite structures-active and passive approach," Composites Science and Technology, vol. 69, no. 11-12, pp. 1678-1685, 2009.

[24] C.-K. Jen, K.-T. Wu, M. Kobayashi, J. H. Kuo, and N. Mrad, "Integrated surface and plate acoustic wave sensors for health monitoring," in Health Monitoring of Structural and Biological Systems, vol. 6532 of Proceedings of SPIE, San Diego, Calif, USA, March 2007.

[25] D. W. Greve, H. Sohn, C. P. Yue, and I. J. Oppenheim, "An inductively coupled lamb wave transducer," IEEE Sensors Journal, vol. 27, no. 2, pp. 295-301, 2007.

[26] K.-T. Wu, W. L. Liu, M. Kobayashi, C.-K. Jen, Y. Ono, and M. Takeuchi, "High temperature guided acoustic wave transducers using mechanical gratings," in Proceedings of IEEE International Ultrasonics Symposium (IUS '09), September 2009.

[27] P. Zheng, D. W. Greve, and I. J. Oppenheim, "Crack detection with wireless inductively-coupled transducers," in Sensors and Smart Structures Technologies for Civil, Mechanical, and Aerospace Systems, vol. 6932 of Proceedings of SPIE, San Diego, Calif, USA, 2008.

[28] X. Zhao, T. Qian, G. Mei et al., "Active health monitoring of an aircraft wing with an embedded piezoelectric sensor/actuator network: II. Wireless approaches," Smart Materials and Structures, vol. 16, no. 4, pp. 1218-1225, 2007.

[29] K.-T. Wu, C.-K. Jen, and N. Mrad, "Non-contact local and global damage detection with integrated ultrasonic transducers," in Sensors and Smart Structures Technologies for Civil, Mechanical, and Aerospace Systems, vol. 6932 of Proceedings of SPIE, San Diego, Calif, USA, 2008.

[30] H. J. Park, H. Sohn, C. B. Yun, J. Chung, and M. Lee, "Development of a non-contact PZT excitation and sensing technology via laser," in Sensors and Smart Structures Technologies for Civil, Mechanical, and Aerospace Systems, vol. 7981, San Diego, Calif, USA, March 2011.

[31] C.-K. Jen, K.-T. Wu, and M. Kobayashi, "Ultrasonic probes having three orthogonal polarizations," IEEE Transactions on Ultrasonics, Ferroelectrics, and Frequency Control, vol. 57, no. 1, pp. 189-192, 2010. 
[32] S. S. Kessler and A. Raghavan, "Co-located triangulation for damage position identification from a single SHM node," in Proceedings of the International Conference on Prognostics and Health Management (PHM '08), Denver, Colo, USA, October 2008.

[33] H. Sohn and S. B. Kim, "Development of dual PZT transducers for reference-free crack detection in thin plate structures," IEEE Transactions on Ultrasonics, Ferroelectrics, and Frequency Control, vol. 57, no. 1, pp. 229-240, 2010.

[34] J.-R. Lee and H. Tsuda, "Sensor application of fibre ultrasonic waveguide," Measurement Science and Technology, vol. 17, no. 4, pp. 645-652, 2006.

[35] N. Mrad, H. Guo, G. Xiao, B. Rocha, and Z. Sun, "On the use of a compact optical fiber sensor system in aircraft structural health monitoring," in Photonic Applications for Aerospace, Transportation, and Harsh Environment III, vol. 8368 of Proceedings of SPIE, Baltimore, Md, USA, 2012.

[36] J.-R. Lee, J. Takatsubo, N. Toyama, and D. H. Kang, "Health monitoring of complex curved structures using an ultrasonic wavefield propagation imaging system," Measurement Science and Technology, vol. 18, no. 12, pp. 3816-3824, 2007.

[37] B. Wang, J. Takatsubo, N. Toyama, and M. Zhao, "The development of laser ultrasonic visualization equipment and its application in nondestructive inspection," in Proceedings of the 17th World Conference on Nondestructive Testing, Shanghai, China, 2008.

[38] C. C. Chia, J.-R. Lee, and H. J. Shin, "Hot target inspection using a welded fibre acoustic wave piezoelectric sensor and a laser-ultrasonic mirror scanner," Measurement Science and Technology, vol. 20, no. 12, Article ID 127003, 2009.

[39] C. C. Chia, H.-M. Jeong, J.-R. Lee, and G. Park, "Composite aircraft debonding visualization by laser ultrasonic scanning excitation and integrated piezoelectric sensing," Structural Control and Health Monitoring, vol. 19, no. 7, pp. 605-620, 2012.

[40] V. V. Kozhushko and H. Krenn, "Detection of laser-induced nanosecond ultrasonic pulses in metals using a pancake coil and a piezoelectric sensor," IEEE Transactions on Ultrasonics, Ferroelectrics, and Frequency Control, vol. 59, no. 6, pp. 12331238, 2012.

[41] F. Yang, D. J. Dorantes-Gonzalez, K. Chen et al., "An integrated laser-induced piezoelectric/differential confocal surface acoustic wave system for measurement of thin film young's modulus," Sensors, vol. 12, no. 9, pp. 12208-12219, 2012.

[42] K.-T. Wu, C.-K. Jen, M. Kobayashi, and A. Blouin, "Integrated piezoelectric ultrasonic receivers for laser ultrasound in nondestructive testing of metals," Journal of Nondestructive Evaluation, vol. 30, no. 1, pp. 1-8, 2011.

[43] J.-R. Lee, C. C. Chia, H. J. Shin, C.-Y. Park, and D. J. Yoon, "Laser ultrasonic propagation imaging method in the frequency domain based on wavelet transformation," Optics and Lasers in Engineering, vol. 49, no. 1, pp. 167-175, 2011.

[44] E. B. Flynn, J.-R. Lee, G. J. Jarmer, and G. Park, "Frequencywavenumber processing of laser-excited guided waves for imaging structural features and defects," in Proceedings of the 6th European Workshop on Structural Health Monitoring, Dresden, Germany, 2012.

[45] S.-C. Hong, J.-R. Lee, S. Y. Chong, C.-Y. Park, and S. Girrens, "Effect of laser pulse fatigue on the mechanical characteristic of an unpainted CFRP plate," in Advances in Structural Health Management and Composite Structures (ASHMCS '12), Jeonju, Republic of Korea, 2012.
[46] H. Gao, B. Lopez, S. M. Ali, J. Flora, and J. Monks, "Inline testing of ERW tubes using ultrasonic guided wave EMATs," in 16th US National Congress of Theoretical and Applied Mechanics (USNCTAM '10), State College, Pa, USA, June-July 2010.

[47] R. Murayama, M. Kobayashi, K.-T. Wu, and C.-K. Jen, "Noncontact ultrasonic sensing using induction based method and integrated piezoelectric ultrasonic transducer," in Proceedings of Symposium on Ultrasonic Electronics, 2010.

[48] H. J. Park, H. Sohn, C. B. Yun, J. Chung, and I. I. B. Kwon, “A wireless guided wave excitation technique based on laser and optoelectronics," Smart Structures and Systems, vol. 6, no. 5-6, pp. 749-765, 2010.

[49] H.-J. Park, H. Sohn, C.-B. Yun, and J. Chung, "Development of a laser-based wireless active sensing technique," in Proceedings of the 6th International Workshop on Advanced Smart Materials and Smart Structures Technology (ANCRiSST '11), Dalian, China, 2011.

[50] H. Sohn, "Laser based structural health monitoring for civil, mechanical and aerospace systems," in Sensors and Smart Structures Technologies for Civil, Mechanical, and Aerospace Systems, vol. 8345 of Proceedings of SPIE, San Diego, Calif, USA, 2012.

[51] H.-J. Park, H. Sohn, C.-B. Yun, J. Chung, and M. M. S. Lee, "Wireless guided wave and impedance measurement using laser and piezoelectric transducers," Smart Materials and Structures, vol. 21, no. 3, Article ID 035029, 2012. 

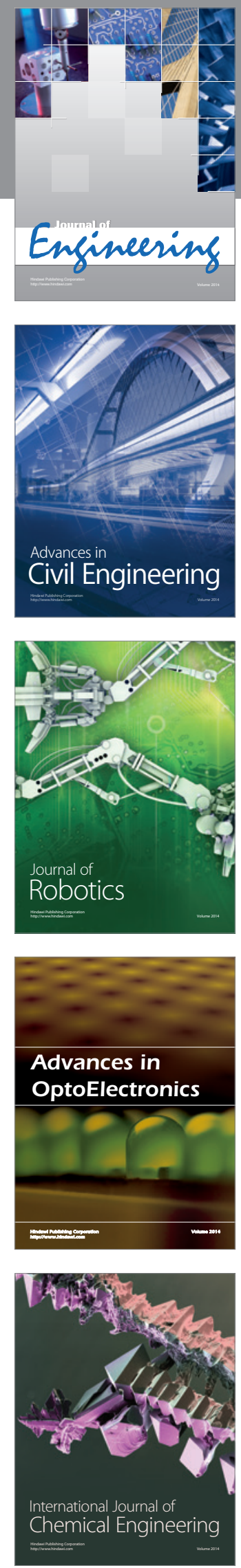

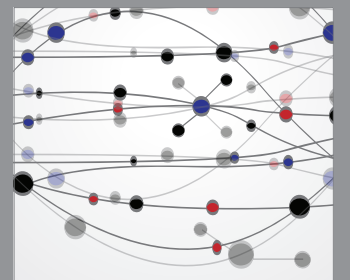

The Scientific World Journal
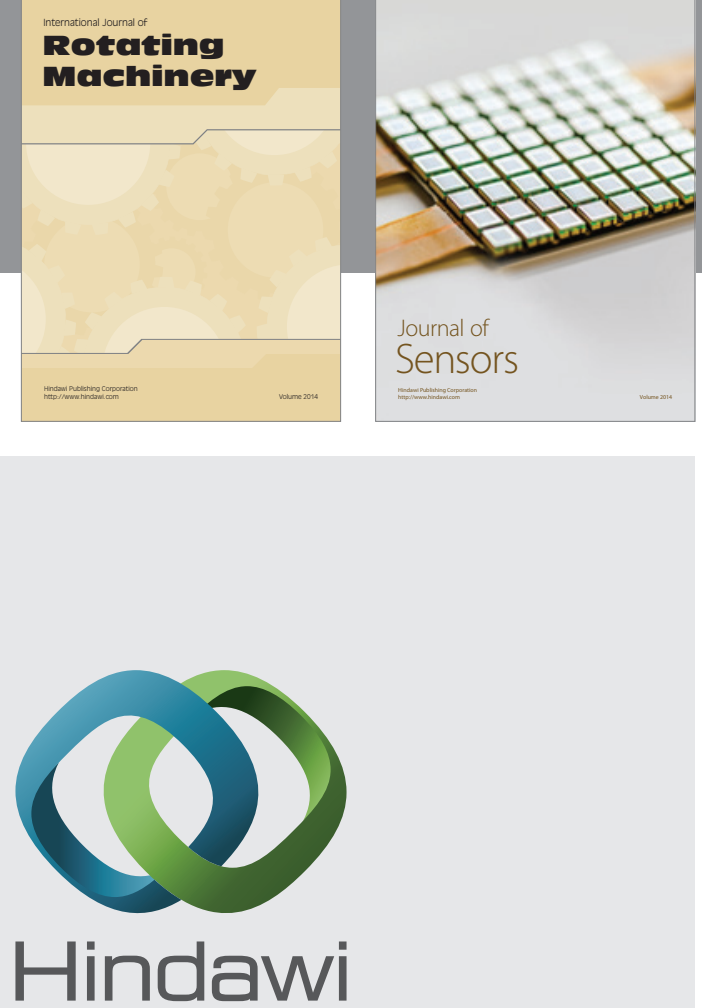

Submit your manuscripts at http://www.hindawi.com
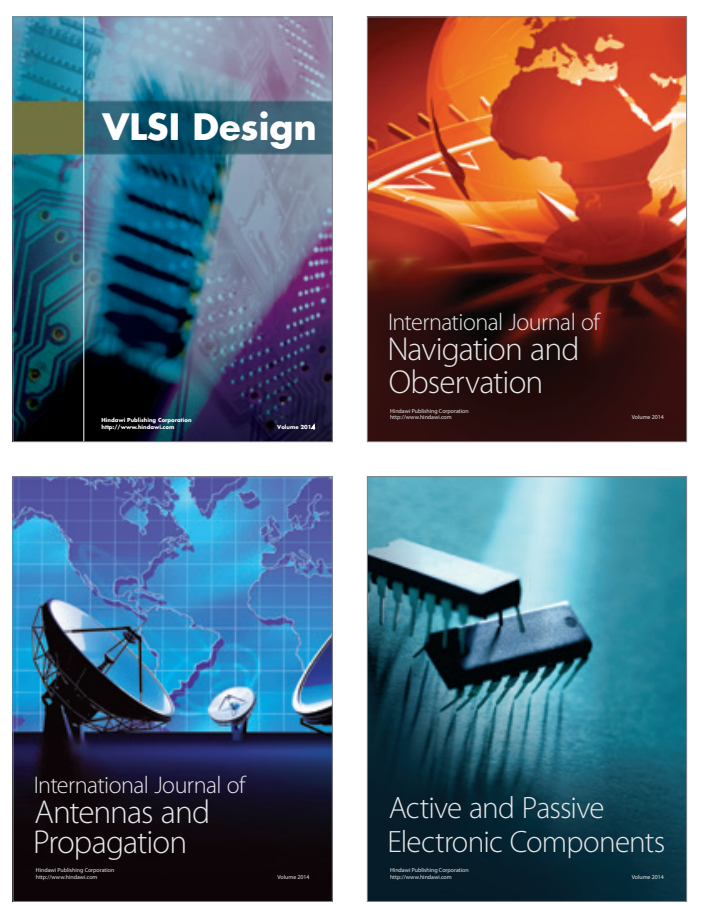
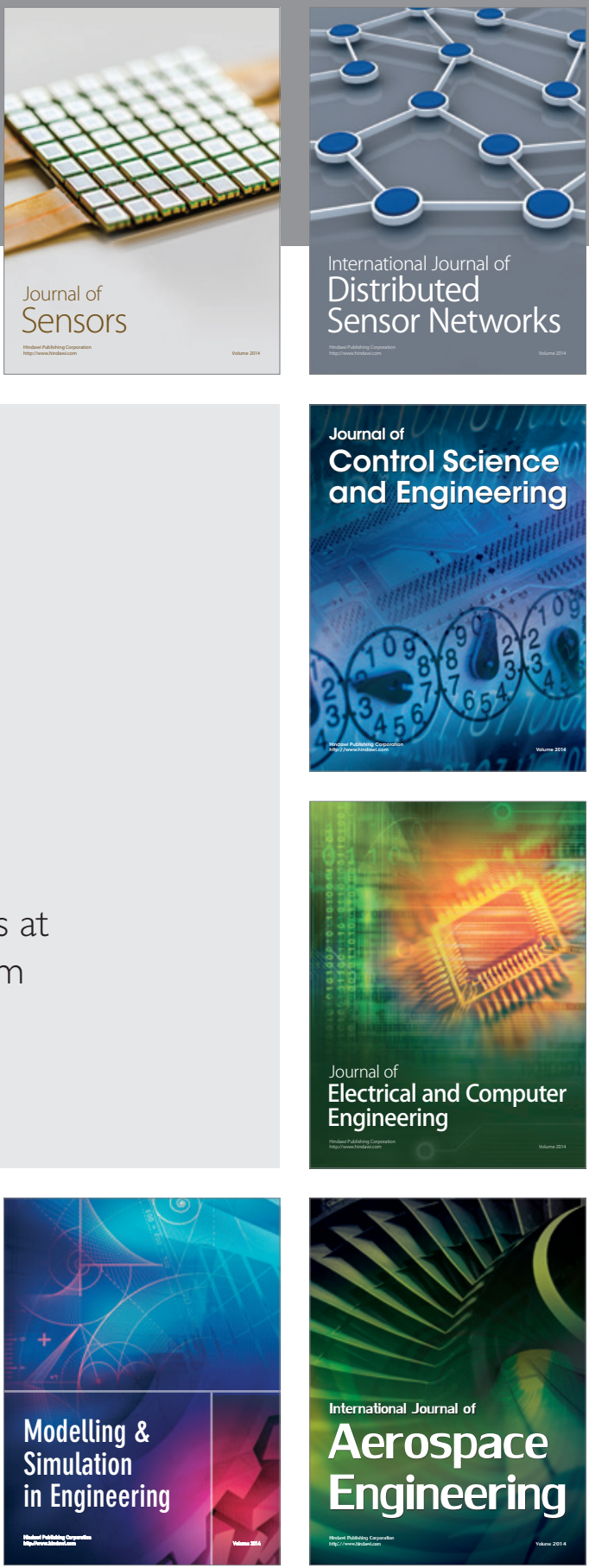

Journal of

Control Science

and Engineering
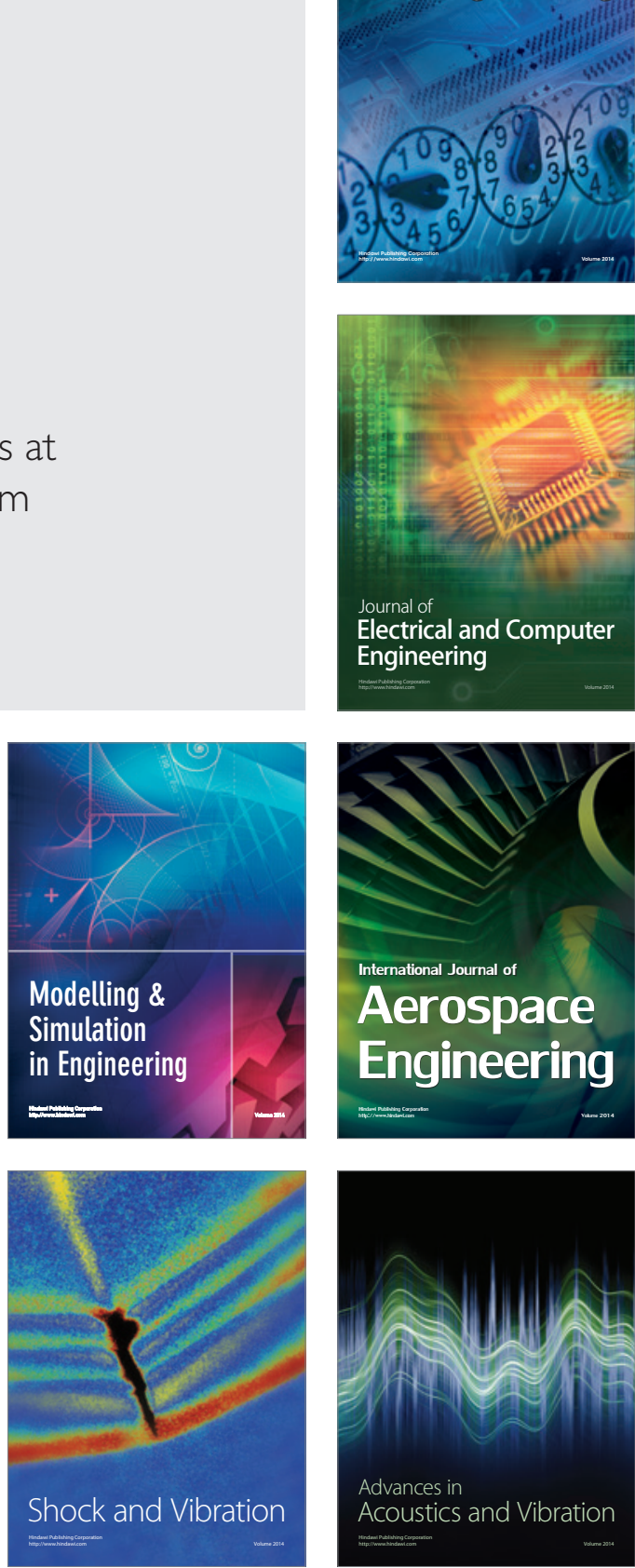